Portland State University

PDXScholar

\title{
An Investigation of the Temporal Relationship Between Agitation and Sleep Disturbances
}

Emily Catherine Denning

Portland State University

Follow this and additional works at: https://pdxscholar.library.pdx.edu/open_access_etds

Part of the Military and Veterans Studies Commons, and the Psychology Commons Let us know how access to this document benefits you.

\section{Recommended Citation}

Denning, Emily Catherine, "An Investigation of the Temporal Relationship Between Agitation and Sleep Disturbances" (2020). Dissertations and Theses. Paper 5585.

https://doi.org/10.15760/etd.7457

This Thesis is brought to you for free and open access. It has been accepted for inclusion in Dissertations and Theses by an authorized administrator of PDXScholar. Please contact us if we can make this document more accessible: pdxscholar@pdx.edu. 
An Investigation of the Temporal Relationship between Agitation and Sleep Disturbances

\title{
by
}

Emily Catherine Denning

\begin{abstract}
A thesis submitted in partial fulfillment of the
\end{abstract} requirements for the degree of

\author{
Master of Science \\ in \\ Psychology
}

Thesis Committee:

Cynthia Mohr, Chair

Todd Bodner

Leslie Hammer

Portland State University

2020 
(C) 2020 Emily Catherine Denning 


\title{
Running Head: AGITATION AND SLEEP DISTURBANCES
}

\begin{abstract}
Suicide rates in the United States have increased almost 30\% since 1999 (Centers for Disease Control and Prevention, 2018), making it the tenth leading cause of death in the country (Xu et al., 2018). This problem is especially prominent for veterans of the U.S. Armed Forces, as veterans comprise $8.5 \%$ of the U.S. population, yet they account for $18 \%$ of all deaths by suicide (Office of Suicide Prevention, 2016). These increasing rates have spurred the need for a better understanding of suicide risk, especially for this highrisk group. Previous research has focused mostly on chronic risk factors, which are useful for identifying who from a population may be more likely to engage in suicidal behaviors, but do not shed light on who may be imminently engaging in suicidal behavior, as would acute warning signs. While it is important to understand what factors affect risk globally, the relationship between more proximal factors needs to be better understood to allow for more targeted and effective risk management. Agitation, a state of mental anguish and emotional overarousal, has emerged as a potential warning sign for suicide (e.g. Rogers, Ringer, \& Joiner, 2016), but the stability of agitation over time has yet to be examined. The present study investigated the stability and temporal relationship between two acute warning signs for suicide, agitation and sleep quality, by testing two cross-lagged panel models. Cross-lagged panel models allow for a test of stability and temporal precedence, a necessary condition to establish causality, between two variables. Temporal precedence can be inferred from a comparison of magnitude of the cross-paths, or the time-lagged paths between the baseline of one variable to the follow-up of the other. This thesis utilized a sample of employed post-9/11 veterans who were all either
\end{abstract}


separated active duty, separated from the National Guard or Reserves, or still active in the National Guard or Reserves. Veterans were recruited from 35 different organizations as part of the larger Study for the Employment Retention of Veterans (SERVe; Hammer et al., 2017). Veterans $(N=497)$ completed the baseline survey and a follow-up survey 3 months later. The sample was mostly white (86\%), male (88.5\%), had been separated from the military and average of 6.20 years $(S D=3.51)$, and most had deployed at least once (87.9\%). Two cross-lagged panel models were computed to investigate the associations between agitation and perceived sleep quality (model 1) and agitation and insomnia symptoms (model 2). Results indicated that agitation was stable over a threemonth period (e.g., model $1, \beta^{*}=.708, S E_{\beta}=.039, p<.001$ ), as were sleep disturbances (e.g., model $1, \beta^{*}=.532, S E_{\beta}=.044, p<.001$ ). Results of two cross-lagged panel models suggested that agitation temporally precedes sleep quality and insomnia symptoms. This finding provides one possible explanation for why veterans experience greater sleep disturbances than their civilian counterparts (Troxel et al., 2015). Additionally, these findings suggest the importance of considering mental health related factors, like agitation, when treating sleep disturbances. One possible implication of these findings is that when veterans seek help for sleep disturbances, this may be used an opportunity to screen for agitation and suicide risk. This may help identify veterans who are at a greater risk for suicide but would not usually seek mental health care for reasons like mental health stigma (Britt et al., 2008). The findings of the present thesis inform future prevention and intervention efforts regarding veteran suicide risk by establishing this temporal association between agitation and sleep disturbances. 


\section{Acknowledgements}

I would like to thank my committee for their time, insights, and support of my thesis. I would like to specifically thank my advisor, Cynthia Mohr, for her encouragement and guidance on this project, as it would not have been possible without her help. I would also like to thank the members of the DYADIC lab and members of my cohort for their enthusiastic support. I would like to thank my family and friends and say a special thank you to my partner, Kristen Denning, for not only emotional support, but also for the countless times she has proofread this document. Additionally, I would also like to acknowledge the SERVe team who made this study possible, the Department of Defense for funding this project, and the veterans and service members for their involvement in the project.

The U.S. Army Medical Research Acquisition Activity, 820 Chandler Street, Fort Detrick MD 21702-5014 is the awarding and administering acquisition office. This work was supported by the Office of the Assistant Secretary of Defense for Health Affairs, through the USAMRMC Broad Agency Announcement under Award No. W81XWH-13-2-0020. Opinions, interpretations, conclusions and recommendations are those of the author and are not necessarily endorsed by the Department of Defense. 


\section{Table of Contents}

Acknowledgments




\section{List of Tables}

Table 1: Descriptives and correlations among main study variables..............................50

Table 2: Comparison of Brief Agitation Measure (BAM) means across studies using the

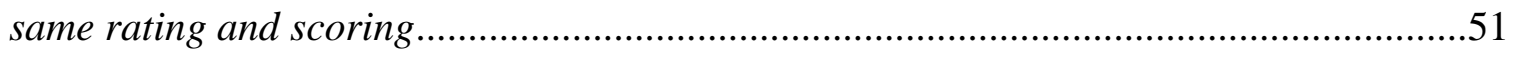

Table 3: Results of cross-lagged panel model of agitation and sleep quality ...................52

Table 4: Results of cross-lagged panel model of agitation and insomnia symptoms ........53 


\section{List of Figures}

Figure 1: Consensus warning signs for suicide developed by a working group from the

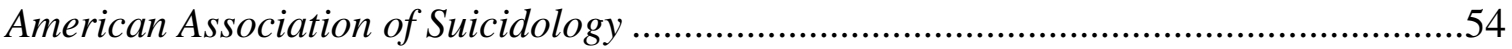

Figure 2: Cross-lagged panel model of agitation and sleep quality ................................55

Figure 3: Cross-lagged panel model of agitation and insomnia symptoms ......................56

Figure 4: Results of cross-lagged panel model of agitation and sleep quality ..................57

Figure 5: Results of cross-lagged panel model of agitation and insomnia symptoms .......58 


\section{Introduction}

Suicide rates in the United States have increased almost 30\% since 1999 (Centers for Disease Control and Prevention, 2018), making it the tenth leading cause of death (Xu et al., 2018). For people ages 10-34, however, suicide is the second leading cause of death, and the fourth leading cause of death for those ages 35-54 (National Institute of Mental Health, 2017). In the year 2016, almost 45,000 people lost their lives to suicide (Centers for Disease Control and Prevention, 2018). This problem is especially salient for

veterans of the U.S. Armed Forces, as their rates of suicide increased 25.9\% from 2005 to 2016 (Office of Mental Health and Suicide Prevention, 2018).

Veterans comprise $8.5 \%$ of the U.S. population, yet they account for $18 \%$ of all deaths by suicide (Office of Suicide Prevention, 2016). Compared to their civilian counterparts, veterans had a 1.5 times greater risk of suicide in 2016 after controlling for gender and age (Office of Mental Health and Suicide Prevention, 2018). These increasing rates have spurred a need for better understanding of suicidal behavior, especially in military populations, with the goal of suicide prevention in mind.

\section{Theoretical Context: The Interpersonal Theory of Suicide}

Early work on suicidality was based in sociological work done by Emile Durkheim. Durkheim (1897) was one of the first theorists to consider causes of suicidality as not only an individual-level problem, but as one influenced by social and societal factors. After considering a wide range of potential factors related to suicide, he found that the social factors he examined had some of the strongest associations to rates of suicide (Durkheim, 1897). Specifically, one indicator of social connection and belongingness emerged as an especially important predictor: social integration. Not only 
does Durkheim describe that a lack of social integration is an antecedent of suicidal behavior, but that a strong social network and strong social connections could also be protective against suicidality (Durkheim, 1897). Durkheim (1897) also noted the connections between degree of integration into society, family, and marriage as predictors of suicide rate. These ideas helped shape and form how suicidality was addressed and also informed future theories and work on suicidality and risk.

Presently, the prevailing theory for understanding, explaining, and predicting suicidal behavior (as based on Durkheim, 1897) is the Interpersonal Theory of Suicide (ITS; Joiner, 2005; Van Orden et al., 2010). The ITS proposes that there are two pathways by which suicide risk can be accumulated - desire for suicide and acquired capability for suicide (Joiner, 2005; Van Orden at el., 2010) - and it is at high levels of both desire and capability for suicide where lethal suicide attempts occur. Desire for suicide is hypothesized to be brought about by high levels of thwarted belongingness and perceived burdensomeness, both related to humans' need to belong being frustrated. Specifically, thwarted belongingness is brought about by a lack of reciprocal close relationships, and perceived burdensomeness is the feeling that one is a burden on close others.

Most individuals who have a desire for suicide, however, do not go on to attempt or die by suicide (Kessler, Berglund, Nock, Wang, \& Page, 2005). Some estimates have found that $3.3 \%$ of Americans seriously consider suicide each year, but only $0.6 \%$ attempt suicide, and only $0.01 \%$ of Americans die by suicide (Kessler et al., 2005). The ITS addresses this fact by proposing that individuals who die by suicide have both a 
higher desire for suicide in addition to an acquired capability for suicide through previous experiences which have increased their tolerance for pain and decreased their fear of death. Some of the experiences that are thought to increase an individual's capability for suicide include combat exposure, previous suicide attempts, childhood maltreatment, and family history of suicide (Joiner, 2005; Van Orden et al., 2010). It is also thought that emotional states characterized by high arousal, such as agitation and impulsivity, are related to overcoming one's fear of death (Ribeiro et al., 2013) and may also be an outcome of extreme feelings of perceived burdensomeness.

Keeping in mind the ways in which the ITS describes how individuals accumulate suicide risk, one can understand why veterans and military-connected individuals may be at increased risk for suicide. The major hypotheses of the ITS have also been evaluated in a number of military samples, and involvement in the military was found to be related to each of the components of the ITS (Selby et al., 2010). In a review of research on the ITS in military service members and veterans, thwarted belongingness, perceived burdensomeness, and acquired capability were all found to be related to suicidal behavior (Selby et al., 2010). Strong relationships with peers and family were found to buffer some of the negative consequences of combat exposure. Different military-related factors, such as number of deployments, combat exposure, or disability resulting from combat, were found to be risk factors (Selby, et al., 2010). Researchers have also investigated the ITS in active duty samples and found that acquired capability of suicide significantly predicted suicidal symptoms for these service members (Bryan et al., 2010). The interaction between perceived burdensomeness and acquired capability for suicide was 
also significant, such that those with the greatest suicidal symptoms were those with high acquired capability for suicide and high burdensomeness (Bryan et al., 2010). Thus, this framework serves as an important lens to view suicidal behaviors in military populations, helping to explain the disproportionate number of deaths by suicide by veterans compared to their civilian counterparts.

\section{Suicide Risk Factors and Warning Signs}

The ITS has helped inform which risk factors are focused on by clinicians, as risk factors can be of practical use for clinicians. These precipitating factors are generally categorized in one of two ways: either chronic or acute. Chronic risk factors are useful for identifying individuals from a population who may be more likely to engage in suicidal behaviors. For example, in most countries in the world, male gender has been found to indicate higher overall risk for suicide, as most people who die by suicide are male (Centers for Disease Control and Prevention, 2018); yet most males do not die by suicide. The same goes for people with mental illnesses like depression and bipolar disorder, which have associations with suicide; yet most individuals who experience depression and bipolar disorder do not go on to die by suicide (Ribeiro et al., 2013). As there is a low base rate for suicide in the population (Kessler et al., 2005), it can be incredibly difficult to accurately assess who will go on to attempt suicide when considering these broad, chronic risk factors alone. What is of more practical use to clinicians is identifying individuals who are at high imminent, or acute, risk of suicidal behaviors by using warning signs. Such acute factors or symptoms are observed in or communicated by an 
individual and indicate a proximal relationship to suicidal behaviors with some amount of empirical support (Ribeiro et al., 2013).

If the goal is accurately assessing suicide risk in order to prevent death by suicide, we need to understand not only which suicide-related factors are warning signs, but also the temporal sequence of warning signs, to allow for prevention efforts to be most successful. One way warning signs have been conceptualized is as a constellation of factors that predict risk, as displayed in Figure 1 (Rudd et al., 2006). Two of the warning signs described and discussed that make up the constellation that precedes suicide attempts, as described by Rudd and colleagues (2006), are agitation and sleep problems, including being unable to sleep or sleeping "all the time," suggesting the importance of future research to consider these factors together. Ribeiro and colleagues (2013) discuss similar factors, which relate to overarousal, including agitation, insomnia, nightmares, and sleep difficulties, as important warning signs to be considered when assessing suicide risk.

A number of obstacles exist that interfere with individuals disclosing their suicidal intent, whether it is to their health providers or close friends and family, which can make traditional approaches to assessing suicide risk problematic. Some research on suicide decedents has found that rates of disclosure of suicide intent to another person are as low as $23.4 \%$ (Choi et al., 2017). Other work conducted a chart review of psychiatric inpatients who had died by suicide found that in their last appointment with their provider, $79 \%$ of patients did not disclose their suicidal intent to their provider (Busch et al., 2003). Estimates based on individuals experiencing suicidal ideation suggest that only 
half of individuals experiencing suicidal ideation disclose their ideation to anyone, whereas $20 \%$ disclosed to friends, family, and healthcare providers, $20 \%$ disclosed only to friends and family, and $6.3 \%$ disclosed only to their healthcare provider (Husky et al., 2016). While people may be hesitant to disclose to their healthcare providers, previous work has demonstrated that the accuracy of the disclosure was highest to providers, and less accurate to friends and family (Hom, Stanley, et al., 2017). The most common barrier to disclosure reported in their sample was concerns regarding stigma (Hom, Stanley, et al., 2017). In other related qualitative work, clinical outpatients who were also experiencing suicidal ideation reported their largest barrier to disclosure was fear of involuntary hospitalization, followed by shame and embarrassment (Blanchard \& Farber, 2018).

Mental health stigma is an especially salient barrier in military populations. Both societal stigma and self-stigma have been found to be related to whether service members receive mental health care (Greene-Shortridge et al., 2007). This stigma may also be an obstacle for disclosure of suicidal intent and risk, especially in populations more likely to stigmatize mental health problems, though it is important to note that mental health stigma has yet to be examined as a barrier specifically to disclosure of suicidal intent in military samples. Even with this in mind, it is still imperative to consider ways of assessing suicidal risk that do not depend fully on a veteran's disclosure of their suicidality.

Previous research notes the importance of warning signs for suicide in risk assessment, but little work has considered the stability of the temporal relationship 
between them. While it is important to understand what factors affect risk globally, the relationships between proximal factors need to be better understood to allow for more targeted and effective risk management. It is also important to consider warning signs that are less obtrusive and less directly related to mental health stigma. Additionally, it is important to investigate the stability of warning signs over time, as very little work has considered the stability of agitation. This thesis, then, focuses on two warning signs of suicidal behavior: agitation and sleep quality.

\section{Agitation}

Definition and Measurement. Agitation is a mental state characterized by mental anguish, turmoil, and emotional overarousal (Ribeiro et al., 2011) and is a useful precursor, or warning sign, to suicidal behavior. Agitation can be identified in someone even when they have a high motivation to conceal their suicidal intentions, as it has little face validity (Ribeiro et al., 2011). Heightened states of emotional arousal have been found to predict when individuals move from thoughts of suicide to engaging in suicide attempts (Nock, Hwang, Sampson, \& Kessler, 2009), as it is thought that agitation can push one to overcome the natural survival instinct that is evolutionarily embedded in humans (Joiner, 2005; Van Orden et al., 2010). Agitated states often are accompanied by outward indications or behaviors, including grimacing or hand-wringing, that may allow for identification without directly asking those in such states. Additionally, agitation is also characterized by internal components such as emotional turmoil and deep discomfort in your "gut" (Chu et al., 2015). 
Agitation and its relationship to suicide have only been researched relatively recently, with studies comprising mostly retrospective chart reviews of suicide decedents. Up until this point, most of the work investigating agitation has been primarily conducted in clinical settings, thus the present section reviews mostly clinical literature considering agitation. In recent work, agitation is often measured in research using the Brief Agitation Measure (BAM; Ribeiro et al., 2011), which is comprised of three items rated on a 7point Likert-type scale including the items: "I want to crawl out of my skin," "I feel so stirred up inside I want to scream," and "I feel a lot of emotional turmoil in my gut." In their process of validating the BAM, Ribeiro and colleagues (2011) found significant relationships between agitation and other related risk factors and warning signs. In a sample of undergraduate students, agitation had a strong association with thoughts of suicide and depressive symptoms, and a moderate association with insomnia severity (Ribeiro, et al., 2011). When investigated in a clinical sample, agitation was found to be related to current suicidality and past suicidal behavior. In addition, strong associations were found between agitation and depressive symptoms, anxiety symptoms, and negative affect, but exploratory factor analyses indicated discriminant validity between agitation and negative affect (Ribeiro et al., 2011).

Other cross-sectional work examining agitation in the context of suicidality has investigated whether there were potential sex differences in the relationship between agitation and suicide-related behaviors (Bryan et al., 2014). Records of 7,698 clinical patients who had been to the Mayo Clinic over a 5-year period were analyzed to test if the relationship between agitation and suicide attempts and ideation was moderated by 
gender. First, results indicated little overlap between agitation and depressive symptoms, suggesting that these are distinct constructs. Regarding gender, agitation was significantly related to suicidal ideation for both men and women (Bryan et al., 2014). Support was also found for gender differences, such that for women no relationship was found between agitation and suicide attempts, however, a significant positive association was found for male patients (Bryan et al., 2014). While this work has found gender differences in this association, it is unclear whether those are a manifestation of the sex differences in suicide attempt rates and suicide death rates or a different factor.

Predictive Validity of Agitation. The only meta-analysis that has investigated the relationship between agitation and suicide behaviors found a moderate, positive association when specifically looking at the outcomes of suicide attempts and death by suicide (Rogers, Ringer, \& Joiner, 2016). Interestingly, this association was not significantly moderated by gender or age. Additionally, agitation had a stronger association with death by suicide than with suicide attempts (Rogers et al., 2016), suggesting it has value as a proximal predictor of suicidal behavior, above predicting thoughts of suicide. One limitation noted by the authors was that all studies included in the meta-analysis were cross-sectional, and they indicated the need for more work investigating how suicide and agitation were associated over time.

One of the methodological challenges to establishing acute correlates of lethal suicidal behavior is the low base rate of suicide deaths in the population, often resulting in inadequate power to investigate individuals who have attempted suicide (Kessler et al., 2005; Van Orden et al., 2010). Some research has addressed this by conducting chart 
reviews and psychological autopsies of suicide decedents, which involves reviewing suicide decedent's medical charts and potentially interviewing family and friends following the decedent's death by suicide. This work has provided support for an association between agitation and death by suicide (Busch et al., 2003; Kovasznay et al., 2004). While there are some methodological limitations to using these methods, chart reviews provide some of the strongest evidence for factors distinguishing between individuals who experience suicidal thoughts but do not attempt, and those who go on to attempt and ultimately die by suicide.

As one example, Busch and colleagues (2003) conducted a chart review of 76 patients who had died by suicide in an attempt to distinguish between acute and chronic risk factors. In their last communication with their psychiatrist, $78 \%$ of patients denied intention for suicidal behaviors, and $34 \%$ of the sample were incorrectly assessed as having low or no risk of suicide, indicating a need for alternate methods of assessing risk than directly asking patients (Busch, Fawcett, \& Jacobs, 2003). It was also found that $50 \%$ of patients demonstrated extreme agitation, as indicated on their charts, thus demonstrating support for agitation as an acute predictor of suicide attempts. Additionally, authors note that due to the nature of retrospectively reviewing patient charts, their estimates of agitation and anxiety may even be underestimates of actual rates (Busch et al., 2003). In another investigation of individuals who had already died by suicide, Kovasznay and colleagues (2004) reviewed psychological autopsies of inmates who had died by suicide. It was also found that the majority (70\%) of inmates had some degree of agitation or anxiety in the period prior to their death, adding more support for 
states of overarousal as acute risk factors for suicide (Kovasznay, Miraglia, Beer, \& Way, 2004). Sani and colleagues (2011) also conducted psychological autopsies of a subset of patients who had died by suicide. Of the 4441 patients who had been hospitalized at an Italian hospital, 96 of them died by suicide over a period of 35 years (Sani et al., 2011). Psychomotor agitation was present in $30.2 \%$ of patients a week prior to their death, and relatedly, inner tension was reported in $54.2 \%$, and racing/crowded thoughts were present in $53.1 \%$.

In sum, there is evidence of the predictive validity of agitation as an acute risk factor for suicidal ideation and suicidal behavior (Busch et al., 2003; Kovasznay et al., 2004). In studies that have analyzed charts or other information on those who have died by suicide, there is much evidence supporting agitation as a preceding factor to a suicide attempt or death by suicide (Busch et al., 2003; Kovasznay et al., 2004; Sani et al., 2011). There are many challenges to predicting suicidal behavior accurately and in a timely way, thus, understanding the warning signs, like agitation, exhibited by individuals who have completed suicide can shed light not only on what predicts suicidal ideation, but also on what leads to individuals attempting and completing suicide.

Agitation in military populations. As previously noted, military-connected individuals have elevated risk of suicidal behaviors (Office of Suicide Prevention, 2018); thus, Rogers and colleagues (2017) examined sex differences and the main components of the ITS in a large military sample. While they did find significant differences in current levels of suicidal ideation, perceived burdensomeness, and thwarted belongingness, such that men had higher levels than women (Rogers et al., 2017), they 
did not find significant differences in agitation or insomnia symptoms. This contradicts Bryan and colleagues' (2014) findings, suggesting military-specific experiences may have an influence on female service member's suicide risk. In another study looking at agitation in a military sample, researchers were interested in the relationship between agitation and an Army soldier's acquired capability for suicide, and how these factors interacted to predict suicidality (Ribeiro et al., 2015). Soldiers were surveyed at an Army recruiting course $(\mathrm{N}=1,208)$, and asked about their current levels of agitation, level of acquired capability for suicide, thoughts of suicide, and current depressive symptoms. Agitation was not a significant predictor of thoughts of suicide, as expected. However, there was a significant interaction between acquired capability for suicide and agitation predicting suicidal risk, such that those with the highest risk were soldiers with high levels of agitation and high levels of acquired capability for suicide (Ribeiro et al., 2015). Results provide support for agitation predicting suicide risk in a cross-sectional study of military-connected individuals, and that other factors, such as one's acquired capability for suicide, may play into the constellation of an individual's risk.

In one of the few studies to my knowledge to investigate the effects of agitation over time, Hom and colleagues (2016) investigated the relationship between agitation, insomnia, and a number of suicide-related factors in a sample of Army recruiter candidates. Agitation, insomnia symptoms, and hopelessness were found to be significant predictors of service members having a mental health visit 18 months later, and insomnia was found to predict depressive symptoms at follow up (Hom et al., 2016). Results 
suggest that agitation may also be an important factor to consider in predicting treatmentseeking behaviors, especially for military-connected individuals.

\section{Sleep Behavior and Suicidality}

Sleep Disturbances and Military Populations: Importance and Definitions.

Another acute warning sign that is important to consider is sleep behavior. In military samples specifically, sleep is an especially important factor to take into consideration, as the post-deployment and reintegration period may present additional stressors that can impact veterans' sleep (Ahern et al., 2015; Bramoweth \& Germain, 2013; Demers, 2011). Some of these include stressors related to family, intimate relationships, work, and identity (Ahern et al., 2015; Demers, 2011). These can contribute to increased prevalence of sleep problems, including poorer sleep quality and reduced sleep duration, that are found in veteran samples (Troxel et al., 2015). With veterans being both at higher risk of sleep difficulties and of suicide-related behaviors, it is important to consider how sleep relates to mental health and suicidality, specifically in this high-risk population.

In the suicidality literature, a number of sleep behaviors have been investigated as potential risk factors. Most work has focused on sleep disturbances, which have been referred to as a "broad class of symptom variables" (Bernert, Kim, Iwata, \& Perlis, 2015, p.15) including insomnia, poor sleep quality, and nightmares (Bernert et al., 2015). Sleep quality, or more specifically subjective sleep quality, is one's overall evaluation about the quality of their sleep (Harvey et al., 2008). Other work has also included insomnia symptoms as indicators of poor sleep quality (e.g. Crain et al., 2014). Sleep disturbances, both as a general measure of sleep, and specific symptoms that comprise sleep 
disturbances (e.g. insomnia and sleep quality) have previously been examined in relation to suicide risk.

Empirical Support for Sleep Disturbances as Predictor of Suicidality. As previously noted, sleep problems have been included as warning signs for suicide (Rudd et al., 2006). A meta-analysis was conducted looking at 18 studies with subjective sleep variables predicting suicide risk, that also included depression as a control variable (Bernert et al., 2015). Poor sleep quality, insomnia, and nightmares, were all found to be significantly related to suicide risk factors, providing empirical support for subjective sleep disturbances as a risk factor for suicide-related behaviors. It is important to note that depression is often controlled for in analyses investigating sleep and suicidality, as depression is "highly intertwined" with both suicidality (Rogers et al., 2018) and sleep disturbances (Alvaro et al., 2013). However, previous work has noted that while depression and suicidality are highly related, they are in fact distinct (Rogers et al., 2018).

Other cross-sectional work has considered this relationship in higher risk samples, including psychiatric outpatients (Bernert et al., 2005) and sleep center patients (Krakow et al., 2011). In a sample of psychiatric outpatients, the relationship between a number of sleep variables and suicidal ideation was investigated while also controlling for other suicide risk factors (Bernert et al., 2005). It was found that insomnia and disturbing dreams/nightmares significantly predicted suicidal ideation, while controlling for breathing problems and depression. Results support that sleep behaviors contribute to suicidality above and beyond their influence on depressive symptoms (Bernert et al., 2005). 
In a large sample of sleep center patients who were seeking treatment for sleeprelated problems, charts were reviewed to investigate associations between sleep problems and suicidal ideation (Krakow et al., 2011). Of the 1,584 patients receiving treatment for sleep problems $13.3 \%$ reported experiencing suicidal ideation in the past 2 weeks. Results indicated significant associations between sleep problems and suicidal ideation, when controlling for levels of depression (Krakow et al., 2011). Specific sleep variables related to suicidality were overall sleep quality, chronic sleep difficulties, and daily fatigue. Other related work has examined this association over longer periods of time. In a study of older adults, with controls matched to a subsample of older adults who had died by suicide and who were part of a larger study that took place over a 10-year period, it was investigated whether earlier sleep disturbances would predict risk of suicide (Bernert et al., 2014). Results indicated that poorer sleep quality at baseline significantly predicted suicide risk at follow-up 10-years later, while controlling for depression (Bernert et al., 2014). These results indicate the importance of sleep quality when evaluating and predicting risk for death by suicide.

Another study aimed to investigate the relationship between sleep disturbances and suicide in a case-control psychological autopsy study of adult suicide decedents in Japan (Kodaka et al., 2014). Suicide decedents were matched with controls, and family members of decedents were interviewed about sociodemographic information, sleep behaviors, and mental health. In $75.5 \%$ of the 52 individuals who had died by suicide, sleep disturbances were found, yet they were only present in $11 \%$ of the controls (Kodaka et al., 2014). Results also indicated that individuals with sleep disturbances were 
estimated to be at 9.7 times greater suicide risk than individuals without sleep disturbances, when adjusting for depressive disorders. Additionally, results indicated that suicide risk was 11.8 times higher for individuals who had been experiencing sleep disturbances for a year or longer (Kodaka et al., 2014). Results support sleep disturbances as risk factors for suicide that can be easily identified by clinicians and physicians.

Sleep and Suicidality in Military Populations. This association has also been demonstrated in military samples. In a chart review of veteran patients seeking treatment for misusing alcohol, it was found that a substantial portion of the sample (38.5\%) reported experiencing suicidal ideation in the past year (Chakravorty et al., 2014). Poor sleep quality, presence of psychiatric disorders, PTSD, older age, and financial insecurity were all significant predictors of suicidal ideation in this sample (Chakravorty et al., 2014). Additionally, five of the veterans had reported attempting suicide in the past year. In another chart review, researchers examined medical charts and clinical notes of 423 veterans who had died by suicide and at some point had utilized VA services, and of those, 90\% had utilized VA services in the year prior to their death (Pigeon et al., 2012). Charts were coded by two reviewers who looked for sleep complaints or disturbances and presence of psychiatric symptoms. When holding age, region, and psychiatric symptoms constant, veterans who had sleep disturbances died more quickly following their last visit to the VA than those without sleep complaints (Pigeon et al., 2012). Authors describe the importance of assessing suicide risk when veterans seek help for sleep problems, as the stigma around mental health in the military is known to interfere with treatment seeking (Britt et al., 2008). 
AGITATION AND SLEEP DISTURBANCES

\section{Agitation and Sleep Examined Together}

A small number of studies have considered agitation and sleep in the same analyses, providing some insight into the relationship between these two phenomena, as well as their association with suicide-related behaviors. As part of a larger study of psychiatric outpatients, the associations between various risk factors for suicide were examined (Rogers \& Joiner, 2018). Agitation was positively correlated with insomnia symptoms, nightmares, and suicidal ideation. Agitation also had moderate positive correlations with feelings of burdensomeness and thwarted belongingness (Rogers \& Joiner, 2018).

Other cross-sectional work has considered both variables as predictors of thoughts of suicide. In a study of 937 military personnel, mostly National Guard members, insomnia symptoms and agitation were investigated as predictors of suicidal ideation (Fisher et al., 2017). Agitation had moderate positive correlations with insomnia, depression, and PTSD symptoms. Analyses investigated whether agitation moderated the relationship between insomnia symptoms and suicidal ideation. It was found that the interaction was significant, and simple slopes revealed that individuals with the highest levels of suicidal ideation were those with the highest levels of agitation and insomnia (Fisher et al., 2017). Additionally, simple slopes indicated that the interaction was only significant at high levels of agitation, but not at mean or low levels of agitation. Authors note that results indicate that both agitation and sleep disturbances play important roles in contributing to suicide risk; however results were based on one time point so the temporal relationship between these two factors is left unclear (Fisher et al., 2017). 
Few studies have examined how agitation and sleep were predictive over multiple time points. One study investigated suicide risk factors as predictors of later mental health visits in a sample of Army recruiters and recruiter candidates (Hom et al., 2016). Participants completed a survey, then medical records were obtained for the following 18 months. The only significant predictors of mental health visits at follow up were agitation, insomnia, and hopelessness, while controlling for baseline depression (Hom et al., 2016). This suggests that sleep difficulties and agitation may be motivators for military-connected individuals to seek treatment, above the effects of depressive symptoms. Additionally, while there may be a number of ways agitation and sleep disturbances have been investigated in the past, future work needs to consider their association independently and in both potential causal directions.

\section{Gaps in the Literature}

Previous work has established an association between agitation and multiple sleep behaviors (Hom, Chu, et al., 2017; Rogers et al., 2017; Rogers \& Joiner, 2018), however, what has been done so far is limited in a number of ways. To further suicide prevention, research needs to elucidate how these warning signs interact over time. To the author's knowledge, the work that has examined agitation and sleep quality together as warning signs for suicide has been exclusively cross-sectional, or with both combined into "overarousal." Additionally, many samples in the literature were clinical samples (Bryan et al., 2014; Ribeiro et al., 2011, 2014; Rogers et al., 2016; Rogers \& Joiner, 2018), or college student samples (Cukrowicz et al., 2006; Hom, Hames, et al., 2017; Ribeiro et al., 2011; Rogers et al., 2016) and it is unclear whether or not results from these studies can 
translate to a non-clinical veteran sample. To better understand acute suicide warning signs, it is important to look at the stability of these over time in a non-clinical, employed sample of veterans to get a better idea of these associations in a more representative veteran sample.

\section{The Present Study}

The present study aimed to consider the stability of two warning signs, agitation and sleep quality, over a three-month period, as well as compare the cross-paths to test if temporal precedence can be established between the two warning signs. Data collected from the Study for the Employment Retention of Veterans (SERVe), which included employed veterans from 35 organizations in the Pacific Northwest was used to investigate these associations. The present study tested the cross-lagged panel models in Figures 2 and 3, thus evaluating the stability and temporal precedence of agitation and sleep quality over a three-month period with the ultimate goal of informing future suicide prevention efforts.

Little, if any work has directly examined agitation and sleep behaviors in the same model. With this in mind, directional hypotheses are inappropriate, as it has yet to be examined if these constructs are stable while controlling for the baseline measure of the other construct. Thus, research questions were examined instead of hypotheses. Specifically, I explored the following research questions:

Research Question 1) Is agitation stable over three months? (path a)

Research Question 2) Is sleep quality stable over three months? (path b) 
AGITATION AND SLEEP DISTURBANCES

Research Question 3) Does baseline agitation predict sleep quality at follow-up?

(path c)

Research Question 4) Does baseline sleep quality predict agitation at follow-up?

(path d)

Research Question 5) How do these cross-paths compare in magnitude? (paths c and d)

Research Question 6) Is there a possibility for a bidirectional relationship? 


\section{Method}

\section{Participants and Procedures}

Participants $(N=497)$ were recruited from 35 organizations across Oregon and Southwest Washington, USA to participate in the Study for the Employment Retention of Veterans (SERVe; Hammer, Wan, Brockwood, Mohr, \& Carlson, 2017). The SERVe project was a Randomized Control Trial (RCT) funded by the Department of Defense that evaluated the effectiveness of a veteran-supportive supervisor training that aimed to train supervisors to better support their veteran employees as they transition to the civilian workforce (VSST; Hammer, Wan, Brockwood, Bodner, \& Mohr, 2019). All participants were currently employed and working at least 20 hours a week. Participants were required to have served in any branch of the US military after 9/11. Veterans completed a baseline survey then a follow-up survey 3 months later, after the implementation of the intervention. All measures were included on both surveys, which were completed online via Qualtrics@2013 (Provo, Utah).

The average age of veterans in the study was 38.4 years $(\mathrm{SD}=9.1)$, and $88.5 \%$ of the sample was male. Most of the participants were married (82\%), white (86\%), and had on average one child $(M=1.17, S D=1.23)$. Most of the participants were separated active duty (47.7\%) or separated from National Guard or Reserves (34.7\%), but a smaller portion were still active in the National Guard or Reserves (17.6\%). Additionally, veterans had been separated from the military and average of 6.20 years $(S D=3.51)$, and most had deployed at least once $(87.9 \%)$. 


\section{Measures}

Demographics and Covariates. Participants reported their age, gender, and marital status. Participants also reported their race and ethnicity, educational attainment, number of children, and other related military-specific demographics, such as branch and number of deployments, which will be probed as potential covariates (see Appendix A for items). As this is part of a larger intervention study, intervention condition was taken into account in analyses, as described in the analysis plan below.

Agitation. The Brief Agitation Measure (BAM; Ribeiro et al., 2011) was used to measure self-reported agitation in the last week. This 3-item measure included the items "I want to crawl out of my skin," "I feel so stirred up inside I want to scream," and "I feel a lot of emotional turmoil in my gut." These items were rated on a 7-point Likert scale ranging from 1 (Not True At All) to 7 (Certainly True). Scores were summed from the three items such that possible scores ranged from 1 to 21 , with higher scores indicating greater agitation. The baseline measure had an alpha of .89, and the 3-month measure had an alpha of .91, indicating good internal reliability. Ribeiro and colleagues (2011) investigated the validity of BAM, which was presented in the introduction of this thesis.

Sleep Quality. Selected items from the Pittsburg Sleep Quality Index (PSQI; Buysse, D.J., Reynolds, C.F., Monk, T.H., Berman, S.R., \& Kupfer, 1998) were used to assess sleep quality. Participants responded on a 4-point Likert-type scale ranging from 1 (Very Bad) to 4 (Very Good) to the items from the PSQI. Subjective sleep quality was measured using one item, "During the PAST 30 DAYS, how would you rate your sleep quality overall?" Additionally, insomnia symptoms was used as an indication of more 
specific aspects of sleep quality with two items from the PSQI (Buysse et al., 1998). An average of the items "During the PAST 30 DAYS, how often could you not get to sleep within 30 minutes?" and "During the PAST 30 DAYS, how often did you wake in the middle of the night or early morning?" have been used as an indicator of insomnia symptoms in previous work (Brossoit et al., 2018; Crain et al., 2014), which inform additional aspects of sleep quality than the overall sleep quality item. 


\section{Data Analysis}

\section{Analysis Plan}

First, preliminary analyses were conducted, including those related to missing

data, distributional assumptions and diagnostics, and descriptive statistics. Then, in order to investigate the research questions presented here, cross-lagged panel models were utilized. Cross-lagged panel models are typically used to evaluate if there is temporal precedence, and potentially causality, when working with non-experimental designs and panel survey data (Kenny, 2005; Kenny \& Harackiewicz, 1979; Newsom, 2016). The main focus of this analysis is the comparison of the standardized cross-lags to evaluate the strength of prediction in helping to determine causality (Kenny \& Harackiewicz, 1979), which in this case allows for a test of this between agitation and sleep quality.

The cross-lagged panel model has two assumptions that need to be met in order to make appropriate conclusions from the model (Kenny, 1975; Kenny \& Harackiewicz, 1979): synchronicity and stationarity. Stationarity assumes that your variables for the same wave were measured at the same time, e.g. baseline measures were both collected from the same baseline survey. The survey design of the present study meets this assumption, as both baseline measures were collected at the same time, as were the follow-up measures. The second assumption, synchronicity, assumes that the magnitude and direction of the associations and causal influences to each variable are stable over time (Kenny, 1975; Kenny \& Harackiewicz, 1979). This also assumes that reliability of measures is consistent across waves, or the comparison of cross-paths may be biased (Kenny, 1975). When the assumptions of synchronicity and stationarity are met, and one 
cross-lag is significantly greater than the other, then one can conclude the comparison of the cross-lags do in fact indicate the existence of a "time-lagged causal effect" (Kenny, 1975). However, if the assumptions are met but both cross-lags are of similar magnitude, then one can conclude the variables in the model have a spurious relationship (Kenny, 1975; Kenny \& Harackiewicz, 1979).

The models tested (see Figures 2 and 3 for conceptual models, Figures 4 and 5 for results) used path analysis structural equation modeling in Mplus (Muthén \& Muthén, 2019). Because data was collected as part of a larger intervention study, it was important to take into account the possible effects of the intervention in the analyses proposed. In order to account for these intervention effects, which occurred between the first and second wave of data collection, standardized residuals were computed in SPSS and used in analyses in Mplus. In order to compute these, simple regression models were computed with each key variable predicted by the intervention condition, and the residuals were saved out as new variables. This allowed for the variables to be standardized when entered into the model, allowing for comparison of cross-lags, as well as remove the variability due to the intervention condition.

Sleep quality and insomnia symptoms were tested separately in cross-lagged panel models with agitation. The base cross-lagged panel model is just identified, as it has zero degrees of freedom with observed variables and only two waves, so information about model fit is not available for the models being tested. First, the stability of each construct is considered with the pathways between the baseline and follow-up measures of the same construct (paths a and b, research questions 1 and 2). Then, standardized 
cross-lagged effects are compared (paths c and d, research question 5) to allow for a comparison in the strength of effect of cross-paths which indicate the potential for causality of one variable over the other.

\section{Preliminary Analyses}

Missing Data: Sleep Quality and Insomnia: One potential concern for the present analyses is that participants who were experiencing poorer sleep quality at baseline may have dropped out of the study before the 3-month follow-up. A dummy variable was created to distinguish those who did or did not complete the 3-month sleep items (all of those missing 3-month sleep quality were also missing the two 3-month insomnia symptom items). A series of logistic regressions were computed to test if any demographic or main study variables predicted if participants did or did not complete the 3-month sleep items. Missing sleep items was predicted significantly by age, military rank, and length in the military, and when testing these predictors in the same model, only age remained significant. Other variables were also tested and were not found to be significant predictors of completing 3-month sleep items, including: BAM, sleep quality, insomnia symptoms, PTSD, combat exposure, hazardous drinking, number of children, number of dependent children, military branch, gender, education, race, and intervention condition. Additionally, differences in those who did or did not complete the 3-month sleep items were also compared in a series of t-tests. Those who did or did not complete the 3-month sleep items did not significantly differ on sleep quality, insomnia symptoms, or $\operatorname{BAM}(p ’ s>.05)$. 
AGITATION AND SLEEP DISTURBANCES

Missing Data: BAM. Similarly, another concern for the present analyses is that participants who were experiencing higher levels of agitation baseline may have dropped out of the study before the 3-month follow-up. Missingness and missingness mechanisms were examined for agitation by creating a dummy variable coded for those who had either completed or did not complete the 3-month BAM. In an additional series of logistic regressions, demographics and main study variables were tested as predictors of missing the 3-month BAM. BAM missingness at 3 months was predicted by age and length of service in the military, which when entered into the same model, only age was a significant predictor. Additional variables were tested and were not significant predictors of missing the 3-month BAM, including: PTSD, hazardous drinking, marital status, number of children, number of dependent children, military branch, number of deployments, combat exposure, gender, education, race, sleep quality, insomnia, or intervention condition.

Unfortunately, missingness of BAM was predicted by BAM baseline scores, indicating that there is an association between levels of agitation and whether or not participants completed the 3-month BAM. In order to further investigate BAM missingness, a t-test was computed to test if there were significant differences on baseline values of BAM for those who completed the baseline and 3-month BAM and those who only completed the baseline BAM. On average, those who did not complete the 3-month BAM had higher levels of agitation at baseline, $M=7.91$, $S D=5.47$, than those who completed both the baseline and 3-month BAM, $M=6.62, S D=4.61$. This difference was significant, $t(492)=2.22, p<.05,95 \% \mathrm{CI}[.15,2.44]$. This indicates that those who 
were lost to follow up were more agitated than those who continued on in the study. Additional t-tests were computed to investigate if these groups of participants significantly differed in their levels of sleep quality and insomnia symptoms, and it was found that they did not significantly differ on their levels of sleep quality or insomnia ( $p$ 's $>.05)$.

Distributional Assumptions and Diagnostics. In order to investigate the distributions of main study variables, histograms and boxplots were created. After initial examination, baseline levels of sleep quality appear to be normally distributed, baseline insomnia symptoms have a slight negative skew, and baseline BAM was the most skewed. After computing descriptive statistics, sleep quality has a skew of -.14 $(S E=.11)$ and kurtosis value of $-.23(S E=.22)$, insomnia symptoms have a skew of -.48 $(S E=.11)$ and kurtosis value of -.43 $(S E=.22)$, and lastly, BAM had a skew value of 1.31 ( $S E=$ $.11)$ and kurtosis value of $.82(S E=.22)$. Curran, West, and Finch (1996) recommend using robust estimators when skew is greater than 2 and kurtosis is greater than 7 , as none of these values surpass these cutoffs, regular maximum likelihood estimation was used. Additionally, boxplots indicated that BAM may have potential outliers (those who report the highest levels of agitation, or those who scored 19, 20, or 21 on the BAM). In order to compute values to test for outliers, simple regression analyses were computed and standardized residuals, mahalanobis distance, and cook's distance were saved out for four models: 1) baseline agitation and sleep quality predicting 3-month agitation, 2) baseline agitation and sleep quality predicting 3-month sleep quality, 3) baseline agitation and insomnia predicting 3-month agitation, and 4) baseline agitation and insomnia 
predicting 3-month insomnia. Together, these four models comprise the same paths being tested in the main cross-lagged models and allow for outliers to be investigated for each path. Standardized residuals were plotted against standardized predicted values and scatterplots were then computed to investigate linearity of associations. Upon inspection, all scatterplots appeared to meet the assumption of linearity. For each of the four models, mahalanobis distances ranges from .01 to 14.97, and cook's distance ranged from 0 to .23. The data were examined to investigate the BAM scores of those with the greatest mahalanobis distance and cook's distance values. The participants who had the greatest mahalanobis distance and cook's distance were those who indicated high BAM scores at baseline or follow-up, ranging from 18 to 21 , but there did not appear to be a relationship with outliers and sleep variables. Additionally, the boxplot of BAM suggested that the same participants' BAM scores were outliers. As agitation is the main focus on the analyses, these participants with the greater levels of agitation, potential outliers, were retained in the dataset to capture the full range of responses to the agitation scale.

Descriptive Statistics. Means, standard deviations, and correlations of main study variables and demographics are displayed in Table 1. Means were also compared to other samples that have used the BAM (Ribeiro et al., 2011) with the same ratings and scoring to better understand the risk of the current sample relative to both military and civilian samples (see Table 2). Compared to other studies using active duty samples who had means that ranged from 4.18 to 4.37 , and standard deviations ranging from 2.50 to 2.56 , our sample has higher means and greater variation, but we are unable to test if these differences are significant. Additionally, our sample has relatively similar mean agitation 
and variability compared to samples of both undergraduates (means ranging from 5.71 to 6.38, standard deviations ranging from 3.67 to 4.81 ) and a sample of psychiatric outpatients $(M=5.77, S D=4.70)$. This comparison of means suggests some veterans may be more agitated than their active duty counterparts, but may have similar levels of agitation of undergraduates and civilian clinical samples, however these are merely comparisons and not significance tests.

Bivariate correlations were computed to probe the associations between the main study variables (see Table 1). Variables were correlated as expected, with higher levels of agitation being associated with poorer sleep quality and more frequent insomnia symptoms at baseline and 3-month. Frequency tables were also computed and indicated that at both waves of data collection responses included all possible values of the BAM, ranging up to 21 at both waves. In fact, at baseline 11 participants indicated the highest rating of agitation (a score of 21). While there are no diagnostic cutoffs for agitation, it is still important to note that there were participants who indicated concerning levels of agitation at both waves, even with the majority of participants indicating low risk.

As participants were recruited from 35 different organizations, there is a potential for clustering. Previous work has indicated that clustering of groups can bias standard errors if ignored (Barcikowski, 1981). In order to investigate the potential effects of clustering by organization, intraclass correlation coefficients (ICC) were computed for the main study variables. The ICC for agitation was .025 , was 0 for sleep quality, and was .02 for insomnia symptoms. Consistent with current organizational research strategies, analyses clustered by organization is not necessary when the ICCs are this small (e.g., 
Lee, McHale, Crouter, Kelly, Buxton. \& Almeida, 2017). The ICC values calculated here are low, thus multilevel approaches were not used in these analyses. 


\section{Results}

\section{Investigation of Specific Research Questions: Cross-Lagged Panel Model}

Two models were tested, one testing the temporal precedence of agitation and

sleep quality, the other testing the temporal precedence of insomnia symptoms and agitation. For both models tested, full information maximum likelihood estimation was used in Mplus version 8 (Muthén \& Muthén, 2019). See Tables 3 and 4 and Figures 4 and 5 for full results of both models.

Research Question 1: Is agitation stable over three months? In order to assess if agitation is stable over 3 months, the pathway between baseline agitation and 3-month agitation was considered (path a on Figures 2 and 3). In both models, baseline agitation was positively associated with 3-month agitation, suggesting that agitation is stable over the 3-month period, even when holding baseline sleep quality constant. Agitation was stable in both the model with sleep quality, $\beta^{*}=.708, S E_{\beta}=.039, p<.001$, and the model with insomnia symptoms, $\beta^{*}=.725, S E_{\beta}=.037, p<.001$. This suggests that agitation is stable over a 3-month period while controlling for levels of sleep quality and insomnia symptoms, such that those who are more agitated at baseline are more likely to be more agitated at follow-up.

Research Question 2: Is sleep quality stable over three months? In order to investigate the stability of sleep quality over three months, the path between baseline sleep quality and 3-month sleep quality was examined (path b from Figure 2), as was the path between baseline insomnia symptoms and 3-month insomnia symptoms (path $\mathrm{b}$ from Figure 3). While holding baseline agitation constant, both sleep quality, $\beta *=.532, S E_{\beta}=$ 
$.044, p<.001$, and insomnia symptoms, $\beta^{*}=.572, S E_{\beta}=.040, p<.001$, were stable over 3 months. This suggests that experiences of poor sleep seem to endure over a three-month period.

Research Question 3: Does baseline agitation predict sleep quality at follow-up? In order to investigate the relationship between baseline agitation and follow-up sleep quality, the results of path c from Figures 2 and 3 are examined. Agitation was significantly predictive of 3-month sleep quality, even when holding baseline sleep quality constant, $\beta *=-0.159, S E_{\beta}=.045, p<.001$. Additionally, the same pattern of results was also found for insomnia symptoms, such that agitation was positively associated with 3-month insomnia symptoms, even above the effects of baseline insomnia symptoms, $\beta *=.169, S E_{\beta}=.041, p<.001$. These findings indicate that greater agitation at baseline was associated with poorer sleep quality and greater insomnia symptoms 3 months later.

\section{Research Question 4: Does baseline sleep quality predict agitation at follow-up?}

In order to test if baseline sleep quality and insomnia predicted follow-up agitation, while controlling for baseline sleep quality, path d from Figures 2 and 3 was examined. Sleep quality negatively predicted 3 -month agitation ${ }^{1}, \beta^{*}=-.082, S E_{\beta}=.038, p<.05$, such that those with greater sleep quality reported lower agitation at follow-up, while controlling for baseline levels of agitation. In contrast with the sleep quality model, insomnia symptoms at baseline were not significantly related to 3-month agitation, while

\footnotetext{
${ }^{1}$ These models were also tested using robust standard errors using the mlr estimator in Mplus. When testing this model with robust estimation, the cross-path between baseline sleep quality and 3-month agitation was no longer significant, but no other results changed. When the insomnia symptoms model was tested with robust estimation, the pattern of results did not change.
} 
controlling for baseline agitation, $\beta^{*}=.056, S E_{\beta}=.036, p=$ n.s. This suggests that insomnia symptoms may function differently than overall sleep quality and are important to consider on their own.

Research Question 5: How do these cross-paths compare in magnitude? In order to investigate this research question, it is necessary to compare the size of the associations between the standardized cross paths, paths $\mathrm{c}$ and $\mathrm{d}$ from Figures 2 and 3. For the sleep quality model, the path between baseline agitation and 3-month sleep quality was larger in magnitude $\left(\beta *=-0.159, S E_{\beta}=.045, p<.001\right)$ than the path between baseline sleep quality and 3-month agitation $\left(\beta^{*}=-.082, S E_{\beta}=.038, p<.05\right)$. This suggests that agitation temporally precedes sleep quality, as these associations are found while controlling for the baseline levels of both agitation and sleep quality. Adding further support, when the sleep quality model was tested using robust estimation the path between baseline sleep quality and 3-month agitation was no longer significant. With robust estimation, all of the other paths remained significant. This suggests that even with a more conservative analysis, agitation temporally precedes sleep quality, but sleep quality does not temporally precede agitation.

For the insomnia symptoms model, the comparison between cross-paths is more straightforward as the path between baseline agitation and 3-month insomnia symptoms was significant and positive $\left(\beta^{*}=.169, S E_{\beta}=.041, p<.001\right)$, whereas the path between baseline insomnia symptoms and 3 -month agitation was not significant $\left(\beta *=.056, S E_{\beta}=\right.$ $.036, p=$ n.s.). This indicates that agitation appears to precede insomnia symptoms, and that feelings of agitation may be contributing to poorer sleep, specifically an increase in 
insomnia symptoms. Findings from both of these models support the notion that feelings of agitation are more strongly related to subsequent sleep quality, compared to the association between sleep quality and subsequent agitation.

Research Question 6: Is there a possibility for a bidirectional relationship?

Results of the previous research question help inform the present question and suggest that the associations between agitation and sleep behavior are not bidirectional. If agitation and sleep quality had a bidirectional association, then it would be expected that both cross-paths would be significant. In the insomnia symptoms model, baseline agitation does not significantly predict 3-month insomnia symptoms $\left(\beta^{*}=.056, S E_{\beta}=\right.$ $.036, p=\mathrm{n} . \mathrm{s}$.), which suggests that the association between agitation and insomnia symptoms specifically is not bidirectional, as only the cross-path between agitation and 3-month insomnia symptoms was significant $\left(\beta^{*}=.169, S E_{\beta}=.041, p<.001\right)$. In the model with sleep quality, there is a chance the associations may be bidirectional, as in the model using regular maximum likelihood estimation, both cross-paths were significant (see Table 3 and Figure 4). However, the association between baseline sleep quality and 3-month agitation was no longer significant when using robust estimation. These findings provide evidence that agitation has temporal precedence over sleep quality and insomnia symptoms, but additional work needs to be done to evaluate the possibility for a bidirectional association. 


\section{Discussion}

When veterans separate from the military, they experience increased stressors related to their family life, intimate relationships, work, and identity (Ahern et al., 2015; Demers, 2011). These stressors may help explain why veterans are not only at higher risk for suicide than their civilian counterparts (Office of Mental Health and Suicide Prevention, 2018), but also experience a greater prevalence of sleep problems (Troxel et al., 2015). The present thesis examined the stability and temporal associations between two suicide warning factors: agitation and sleep quality. These factors have previously been identified as acute warning signs for suicide (e.g. Rudd et al., 2006), but their stability and temporal associations have yet to be examined. Up until this point, little, if any work has examined these warning signs in the same model, thus the results of the present thesis are especially illuminating as they provide evidence for the stability of both agitation and sleep disturbances over a 3-month period in a military sample. These findings have noteworthy implications for suicide prevention strategies with veterans.

\section{Stability of Agitation and Sleep Disturbances}

Findings of the present thesis provide support for the stability of both agitation and sleep disturbances, specifically perceived sleep quality and insomnia symptoms. In both cross-lagged panel models tested, baseline agitation was positively associated with 3-month agitation, while controlling for baseline sleep. Additionally, sleep quality was stable when accounting for agitation, and in a separate model, insomnia symptoms were also stable while holding baseline agitation constant. These results suggest that if a veteran is experiencing high levels of agitation, they are likely to continue to report high 
agitation levels three months later. This has important implications for intervention, as this supports the persistence of these emotional states like agitation over a three-month period. Additionally, if agitation is stable over longer periods of time, this may suggest agitation may only function as an acute warning sign in the presence of other factors that were not included in the present model. Future work should investigate if agitation is stable over longer periods of time in order to better inform intervention and prevention.

These findings have potential implications for suicide prevention and intervention. Agitation is considered an acute warning sign (Rudd et al., 2006), as it is thought to be part of the constellation of factors that predict engagement in suiciderelated behavior. With that in mind, it is especially interesting that our evidence suggests there is stability over a 3-month period. Agitation may be used as an early step to assess risk without the need to explicitly mention suicide, as is typical of suicide risk assessment. If agitation is stable over a 3-month period, that also suggests that there is at least a 3-month window of time to identify at-risk individuals and intervene.

Additionally, unrelated to suicide risk, agitation on its own is not a pleasant state in which to be, especially for a 3-month duration, or potentially longer. If agitation persists for this amount of time, and as the findings of the present study indicate it is related to poorer sleep and greater incidence of insomnia symptoms, there may be other downstream effects that could be detrimental to one's health and well-being in addition to the experience of agitation itself. While agitation is prevalent in individuals who have died by suicide in the period preceding their death (e.g. Busch et al., 2003), less work has examined other potential outcomes of agitation, so it is unclear what else agitation may 
lead to. Previous cross-sectional work has found associations between agitation and suicidal ideation, feelings of burdensomeness, and thwarted belongingness (Rogers \& Joiner, 2018). Other work has found associations between agitation, depression, and PTSD symptoms (Fisher et al., 2017). Additionally, agitation was found to be predictive of mental health visits (Hom et al., 2016). Building upon the previous that has investigated agitation, the evidence of the present study suggests sleep disturbances may be an outcome of agitation. Sleep disturbances have been shown to be related to hypertension (Gottlieb et al., 2006), diabetes (Resnick et al., 2003), and mortality risk in older adults (Dew et al., 2003). Poor sleep for a long period of time has the potential to reduce social functioning, and in addition, poor sleep has been demonstrated to be associated with loneliness (Cacioppo et al., 2002), which could potentially exacerbate suicide risk according to the ITS (Joiner, 2005; Van Orden et al., 2010). The stability of both sleep disturbances and agitation and important to consider, as well as the downstream effects of prolonged agitation and poor sleep.

\section{Comparison of Over Time Associations Between Agitation and Sleep}

The main focus of a cross-lagged panel analysis is on the comparison of the crosspaths, or path c and d in Figures 2 and 3. In the present analysis, findings indicated that agitation temporally precedes sleep quality. Specifically, there was support for the temporal association between baseline agitation and 3-month sleep quality and between baseline agitation and 3-month insomnia symptoms. At the same time, there was far less support for the association between baseline sleep quality and 3-month agitation, and the path between insomnia symptoms and 3-month agitation was not significant. Comparison 
of the magnitude of these paths, as well as their significance, provides support for the temporal precedence of agitation occurring before subsequent reduced sleep quality and increased insomnia symptoms. The results of the insomnia symptoms model allow us to conclude that agitation temporally precedes insomnia symptoms, as one cross-path was significant while the other was not, which helps elucidate the nature of the agitation-sleep association. In the sleep quality model, the path between baseline sleep quality and 3month agitation was significant, but smaller in magnitude when using regular maximum likelihood estimation. The significance of that path changed when using robust estimation, however, indicating that with more conservative standard errors this path is of smaller magnitude than that of the cross-path between baseline agitation and 3-month sleep quality.

These findings are important as they do not provide evidence for a bidirectional association, or feedback cycle between experiences of agitation and poor sleep. This suggests it is possible the cause of the agitation is not poor sleep, but likely more related to one's mental health and suicidality. The ITS (Joiner, 2005; Van Orden et al., 2010) suggests that a number of factors interact to predict suicide risk, including factors specifically related to social relationships. It is possible that social factors, like feelings of burdensomeness or thwarted belongingness could possibly be related to both agitation levels and quality of sleep. It is important to consider not only one warning sign on its own but consider them in the context of the constellation of warning signs that could be present. Future work should consider other factors that could amplify the effects of agitation, like social withdrawal. The findings of the present thesis also provide some 
evidence for the validity of agitation, specifically the BAM (Ribeiro et al., 2011), as it is clearly distinct from physical health related emotional responses. Additionally, this provides evidence for one of the potential outcomes of experiencing agitation: poorer sleep health. This has important implications for mental health providers, as it is likely that their more agitated patients are also experiencing a reduction in sleep quality. It is important to note that our sample was not a clinical sample, so more work should be done considering the outcomes of agitation in high risk clinical samples.

\section{Possibility of Bidirectionality}

While the present findings do not provide support for a bidirectional association between agitation and sleep quality (agitation appears to temporally precede sleep quality, not the other way around), that does not necessarily mean they do not have some amount of feedback to each other as people do not experience life in a vacuum. Previous work has found bidirectional associations between sleep disturbances and both depression and anxiety (Alvaro et al., 2013) thus it appears that agitation may be distinct from anxiety and depression, although still related. It is important for future work to continue considering the unique role agitation plays in affecting sleep and other health behaviors. It is also possible that this association could be bidirectional on a daily level, as opposed to over a three-month span, as it is more likely your sleep will be impacted by your emotions when they are more temporally proximal. These findings suggest that the period of time examined is especially important to consider, and future work should look both at daily associations, as well as spans of time longer than three months. 


\section{Implications}

The findings of the present study have important implications for suicide prevention and improving sleep health of veterans. One of the strengths of utilizing a more discreet risk assessment for veterans, such as the BAM (Ribeiro et al., 2011), is being able to investigate risk without triggering stigma related to mental health care. Mental health stigma is an especially salient obstacle for veterans seeking help for mental health problems (Greene-Shortridge et al., 2007). Many military-connected individuals are far less likely to seek help for a mental health problem than they are a physical health problem (Britt, 2000), and research supports stigma as a barrier to help seeking in military populations (Greene-Shortridge et al., 2007). For example, one study of Army combat soldiers found that of those who screened positively for a mental disorder, only 23 to 40 percent actually sought out care for that disorder (Hoge et al., 2004). Additionally, those with mental disorders also reported greater concern about stigmatization around mental health and seeking mental health care (Hoge et al., 2004). With this in mind, an approach to assessing suicide risk in military-connected individuals that can avoid the stigma of seeking help for mental health problems could potentially target individuals who would not otherwise seek care.

As evidence suggests agitation temporally precedes sleep disturbances, one potential implication of this finding is that if a veteran seeks help for sleep problems, one possible reason for those is agitation or their underlying mental health. One possible strategy that follows from this association is that the BAM can be administered as a discreet way of assessing suicide risk such that those who may otherwise go under the 
radar can receive the help they need. One previous study of general practitioners found that while providers may be aware of general risk factors, they are less knowledgeable about acute warning signs (Michail et al., 2017). If medical doctors can be more informed about the connections between warning signs, such as sleep health and suicide risk, then there is a potential for more high-risk individuals to be identified and given the care and resources they need. Medical professionals who are more knowledgeable about suicide also report more positive attitudes towards suicide, and greater confidence in how to handle working with suicidal patients (Boukouvalas et al., 2019). We also know that suicide decedents generally have low rates of disclosure of suicidal intent (Choi et al., 2017), but when they do disclose, they are most accurate when they disclose to their medical provider (Hom et al., 2017). Previous work has suggested that health care staff that have received some kind of suicide prevention gatekeeper training (e.g. ASIST, QPR) report greater knowledge and confidence working with suicidal patients (Silva et al., 2016). Unfortunately, other work has also indicated that most participants (52.9\%) in a large study of professionals working in behavioral health care had not received suicide prevention or assessment training (Silva et al., 2016). On a more positive note, however, medical professionals are likely to be open to receiving training regarding suicide risk, as one study of general practitioners found that the majority of their sample indicated interest in receiving suicide prevention training (Michail et al., 2017). Medical providers could play an important role in suicide prevention for these reasons, thus it is especially important for them to receive more training on the recognition of warning signs for suicide. 
AGITATION AND SLEEP DISTURBANCES

These findings also have implications for medical professionals and other individuals who work with veterans specifically. Previous work has indicated that veterans with sleep disturbances are at higher risk for suicide (Pigeon et al., 2012). Thus, it is crucial that those who interact with veterans take sleep complaints seriously and understand the implications for suicide risk. Those who work with veterans and militaryconnected individuals can note when sleep disturbances arise and use them as an opportunity to check-in with the veteran about the state of their mental health, their thoughts of death, access to lethal means such as firearms, and an opportunity to provide extra support and connect them to resources. It is important to do so while also being sensitive to the stigma they may have associated with mental illness that has been shown to be a barrier to veterans seeking care (Greene-Shortridge et al., 2007). Addressing mental health stigma is an important component of preventing suicide for veterans specifically. It is also possible that traditional methods of trying to treat sleep problems (e.g. improving sleep hygiene, prescribing sleep medications) may not adequately address the underlying problem, which could be related to one's underlying mental health challenges. Primary care providers working with veterans can benefit from considering the number of potential causes of sleep problems that relate to social and emotional functioning, as merely prescribing a medication and moving on may divert veterans from their pathway of care that would lead to their mental health being addressed.

Findings of the present thesis also have implications for clinical psychologists and mental health practitioners, as it is likely the patients they are seeing who are experiencing mental health challenges may also be experiencing poor sleep quality. 
Previous work has also indicated that levels of agitation were predictive of subsequent mental health care visits in a sample of Army recruiter and recruiter candidates (Hom et al., 2016). Because the results of the present study indicate that agitation is predictive of sleep quality and insomnia symptoms, mental health care providers can include assistance with health behaviors like sleep into their plan of care. It is important for mental health practitioners to consider the interplay between one's physical and mental health.

Additionally, these findings have implications for interventions, both those related to sleep and suicide, as this pattern of results indicates an intervention to improve sleep would not have an impact on agitation. However, the efficacy of one's sleep intervention may depend on the mental health issues held by those the intervention is targeting. In terms of suicide preventions, this provides some information around the stability of agitation, indicating that there is likely to be a window of opportunity for at least a few months to intervene on agitated individuals. Additionally, this study has implications for supervisor interventions, like SERVe, such that supervisors of veterans can be better educated about suicide warning signs and the interplay between them. Supervisors may be able to better support their veteran employees if they have a better understanding of their suicide risk, and of common warning signs and risk factors.

\section{Limitations}

The present study is not without limitations. When utilizing a cross-lagged approach to investigating temporal associations, one possible problem is having the incorrect amount of time between the baseline and follow-up data collections. It is possible that the lag between baseline and follow-up was incorrect and the cross-lagged estimates may 
have been biased by this (Newsom, 2016). The effects of poor sleep may be better measured at shorter intervals than three months, perhaps at a daily or weekly level. It is possible that at shorter intervals there is a bidirectional relationship, but that agitation is the driver over longer periods of time, like the three-month interval examined in the present study. Previous research investigating the effects of life events on subjective well-being (SWB) found that individuals return to their baseline SWB within three months (Suh, Diener, \& Fujita, 1996), thus it is possible the lag in the proposed analyses was appropriate. Future work can consider investigating these associations over different periods of time to provide support for stability over various periods of time.

Additionally, participants who dropped out after the baseline data collection had significantly greater levels of agitation than those who remained in the study for followup. It is possible that the results may have been impacted by a restricted range in the follow-up, as those who were of greater risk were more likely to have a missing followup score. The follow-up BAM scores did have the same range as the baseline, but it is important to interpret results with this in mind, as it is likely levels of agitation were related to missingness. It is also possible that the participants who dropped out of the study may have had less stable agitation levels over time, which suggests that our estimate of stability for agitation may be overinflated, as individuals with more stable levels of agitation were potentially overrepresented in this sample, thus the stability of agitation should be interpreted with this limitation in mind as well. Furthermore, another limitation is the use of agitation as an indicator of suicide risk. Although there is empirical support for the use of BAM for this purpose (Ribeiro et al., 2011), it is also 
possible that individuals could report being agitated for other reasons than suicidality as the base rate for suicide is low in the population (Kessler et al., 2005). In our larger study we did not directly measure suicidality or suicidal ideation, so we are unable to know the exact level of suicide risk of our participants at the time of data collection. That being said, agitation is still an important aspect of suicidality to study on its own.

Finally, the sample being used is a majority male sample, which may reflect the overall gender breakdown of U.S. Veterans (Department of Defense, 2017), but makes it difficult to examine the effects of gender in the present analyses. Female veterans also have especially high rates of suicide compared to their civilian counterparts (Office of Suicide Prevention, 2016). For example, in 2014 the Office of Suicide Prevention at the VA reported that male veterans had a 19 percent higher rate of suicide than male civilians while female veterans had a 250 percent higher rate than their civilian counterparts. Although women may make up a smaller portion of U.S. veterans, it is especially important that future research focuses on the effects gender might play in veteran suicide risk.

\section{Future Directions}

In order to best inform prevention and future interventions, it is important to consider the association between agitation and sleep while also examining additional mediators and moderators. An important next step in the research investigating agitation is better understanding the influence of other relevant social factors as suggested by the ITS (Joiner, 2005; Van Orden et al., 2010). Social relationships have also been associated with sleep quality (Kent et al., 2015), thus the association between these is especially 
important to consider in a social context. Some social factors may serve as protective factors and may buffer the association between agitation and sleep quality. Social support has been associated with lower suicide risk in previous work with veterans (Pietrzak et al., 2010) and may potentially moderate the association between agitation and sleep quality, and is important to consider in future work. The ITS (Joiner, 2005; Van Orden et al., 2010) proposes that part of what allows individuals to overcome their fear of death are states of high arousal, like agitation. It is important, then, for future research to include aspects of acquired capability for suicide, including one's fearlessness about death, to better understand the specific association between agitation and capability for suicide.

There may be potential mechanisms that are linking agitation to subsequent poor sleep quality, such as a reduction in sleep hygiene, that are important for future work to consider, in addition to other mechanisms by which agitation may be related to health behavior changes, that could potentially have negative downstream effects on one's physical health. Additionally, future work can consider other military-related factors that have been shown to be related to suicide risk for veterans such as number of deployments and combat exposure (Selby et al., 2010) and how they relate to sleep quality and agitation. We know veterans experience greater sleep problems than their civilian counterparts (Troxel et al., 2015), thus it is important to understand the factors that may confer this greater incidence of sleep disturbances in addition to suicide. 


\section{Conclusion}

Veteran suicide rates are 1.5 times higher than that of their civilian counterparts, while controlling for age and gender (Office of Mental Health and Suicide Prevention, 2018). Research has focused on better understanding chronic risk factors, but less work has focused on acute risk factors or warning signs. Additionally, veterans also have a greater degree of sleep disturbances (Troxel et al., 2015) and it is important for work to consider the possibility of agitation or suicide-related factors as potential contributors to the greater prevalence of sleep disturbances in veterans. This thesis specifically tested the stability and temporal associations between two suicide warning signs: agitation and sleep disturbances using two cross-lagged panel models. Firstly, results suggest that agitation and sleep disturbances were stable over three months. Additionally, results indicated that the association between baseline agitation and 3-month sleep quality was stronger in magnitude than the association between baseline sleep quality and 3-month agitation, providing evidence that agitation temporally precedes sleep disturbances. This illuminates the effect that one's mental health, specifically levels of agitation, can potentially have on sleep disturbances. These findings provide support for increased access to mental health care and resources for veterans, as we can see that helping reduce agitation may also help improve their sleep problems. Additionally, providers who work with veterans can utilize sleep complaints as an opportunity to assess suicide risk, potentially capturing higher risk veterans who may have otherwise gone under the radar. Lastly, these findings did not support the possibility of a bidirectional association between these variables. Future research will benefit from testing different time lags and 
from considering how social factors may influence this association, especially those related to the Interpersonal Theory of Suicide (Joiner, 2005; Van Orden et al., 2010). Ultimately, this proposed study can inform current suicide prevention efforts and inform alternate approaches to suicide risk assessments that could allow for more high-risk veterans to be identified as their sleep problems arise, potentially reducing the number of veteran suicide deaths that occur. 
AGITATION AND SLEEP DISTURBANCES

Tables

Table 1. Descriptives and correlations among main study variables.

\begin{tabular}{llccccccc}
\hline \multicolumn{1}{c}{ Variable } & $\mathrm{M}$ & $\mathrm{SD}$ & 1. & 2. & 3. & 4. & 5. \\
\hline 1. & BL Agitation & 6.83 & 4.79 & -- & & & & \\
2. & 3-mo Agitation & 6.31 & 4.45 & $.72 * * *$ & -- & & & \\
3. & BL Sleep Quality & 2.49 & 0.69 & $-.45 * * *$ & $-.38 * * *$ & -- & & \\
4. & 3-mo Sleep Quality & 2.56 & 0.68 & $-.38 * * *$ & $-.44 * * *$ & $.59 * * *$ & -- & \\
5. & BL Insomnia Symptoms & 3.02 & 0.77 & $.28 * * *$ & $.28 * * *$ & $-.42 * * *$ & $-.40 * * *$ & -- \\
6. & 3-mo Insomnia Symptoms & 2.97 & 0.79 & $.34 * * *$ & $.35 * * *$ & $-.40 * * *$ & $-.45 * * *$ & $.63 * * *$ \\
\hline
\end{tabular}

Note. $\mathrm{BL}=$ baseline data collection, $3-\mathrm{mo}=3$-month data collection. $* * * p<.001$. 
AGITATION AND SLEEP DISTURBANCES

Table 2. Comparison of Brief Agitation Measure (BAM) means across studies using the same rating and scoring.

\begin{tabular}{|c|c|c|c|}
\hline Sample & BAM Range & BAM Mean & SD \\
\hline SERVe Baseline $(N=497)$ & $3-21$ & 6.83 & 4.79 \\
\hline SERVe 3-Month $(N=415)$ & $3-21$ & 6.31 & 4.45 \\
\hline Army Recruiters $^{1}(\mathrm{~N}=1,208)$ & -- & 4.35 & 2.50 \\
\hline Army National Guard SMs ${ }^{2}(\mathrm{~N}=327)$ & $3-21$ & 5.26 & 3.77 \\
\hline Army Recruiters ${ }^{3}(\mathrm{~N}=2,596)$ & $3-21$ & 4.37 & 2.56 \\
\hline Army Recruiters $^{4}(\mathrm{~N}=3,387)$ & -- & 4.32 & 2.50 \\
\hline Male Army SMs ${ }^{5}$ & -- & 4.34 & 2.51 \\
\hline Female Army SMs ${ }^{5}$ & -- & 4.18 & 2.40 \\
\hline Undergraduates ${ }^{6}(\mathrm{~N}=212)$ & -- & 5.71 & 3.67 \\
\hline Psychiatric Outpatients $^{6}(\mathrm{~N}=107)$ & -- & 5.77 & 4.70 \\
\hline Undergraduates $^{7}(\mathrm{~N}=186)$ & $3-21$ & 6.38 & 4.81 \\
\hline
\end{tabular}

Note. SERVe baseline and 3-month are from the present study. ${ }^{1}$ Ribeiro et al., 2015; ${ }^{2}$ Turnell et al., 2019; ${ }^{3}$ Hom et al., 2016; ${ }^{4}$ Silva et al., 2017; ${ }^{5}$ Rogers et al., 2017; ${ }^{6}$ Ribeiro et al., 2011; ${ }^{7}$ Rogers et al., 2016. 
Table 3. Results of cross-lagged panel model of agitation and sleep quality.

\begin{tabular}{|l|c|c|c|c|}
\hline \multirow{2}{*}{} & \multicolumn{2}{|c|}{3 -Month Agitation } & \multicolumn{2}{c|}{3 -Month Sleep Quality } \\
\cline { 2 - 5 } & $\beta^{*}$ & SE & $\beta^{*}$ & SE \\
\hline Baseline Agitation & $.708^{* * *}$ & .039 & $-.159 * * *$ & .045 \\
\hline $\begin{array}{l}\text { Baseline Sleep } \\
\text { Quality }\end{array}$ & $-.082^{*}$ & .038 & $.532^{* * *}$ & .044 \\
\hline
\end{tabular}

Note. $* p<.05, * * * p<.001$ 
Table 4. Results of cross-lagged panel model of agitation and insomnia symptoms.

\begin{tabular}{|l|c|c|c|c|}
\hline \multirow{2}{*}{} & \multicolumn{2}{|c|}{3 -Month Agitation } & \multicolumn{2}{c|}{ 3-Month Insomnia Symptoms } \\
\cline { 2 - 5 } & $\beta^{*}$ & $\mathrm{SE}$ & $\beta^{*}$ & $\mathrm{SE}$ \\
\hline Baseline Agitation & $.725^{* * *}$ & .037 & $.169^{* * *}$ & .041 \\
\hline $\begin{array}{l}\text { Baseline Insomnia } \\
\text { Symptoms }\end{array}$ & .056 & .036 & $.572^{* * *}$ & .040 \\
\hline
\end{tabular}

Note. $* * * p<.001$ 


\section{Figures}

\section{Consensus Warning Signs for Suicide}

Are you or someone you love at risk for suicide? Get the facts and take action.

Call 9-1-1 or seek immediate help from a mental health provider when you hear, say or see any one of these behaviors:

- Someone threatening to hurt or kill themselves

- Someone looking for ways to kill themselves: seeking access to pills, weapons, or other means

- Someone talking or writing about death, dying, or suicide

Seek help by contacting a mental health professional or calling 1-800-273TALK for a referral should you witness, hear, or see anyone exhibiting any one or more of these behaviors:

- Hopelessness

- Rage, anger, seeking revenge

- Acting reckless or engaging in risky activities, seemingly without thinking

- Feeling trapped-like there's no way out

- Increasing alcohol or drug use

- Withdrawing from friends, family, or society

- Anxiety, agitation, unable to sleep, or sleeping all the time

- Dramatic changes in mood

- No reason for living; no sense of purpose in life

Figure 1. Consensus Warning Signs for Suicide developed by a working group from the American Association of Suicidology.

Rudd, D. M., Berman, A. L., Joiner, T. E., Nock, M. K., Silverman, M. M., Mandrusiak, M., ... Witte, T. (2006). Warning signs for suicide: Theory, research, and clinical applications. Suicide and Life-Threatening Behavior, 36(3), 255-262.

https://doi.org/10.1521/suli.2006.36.3.255 


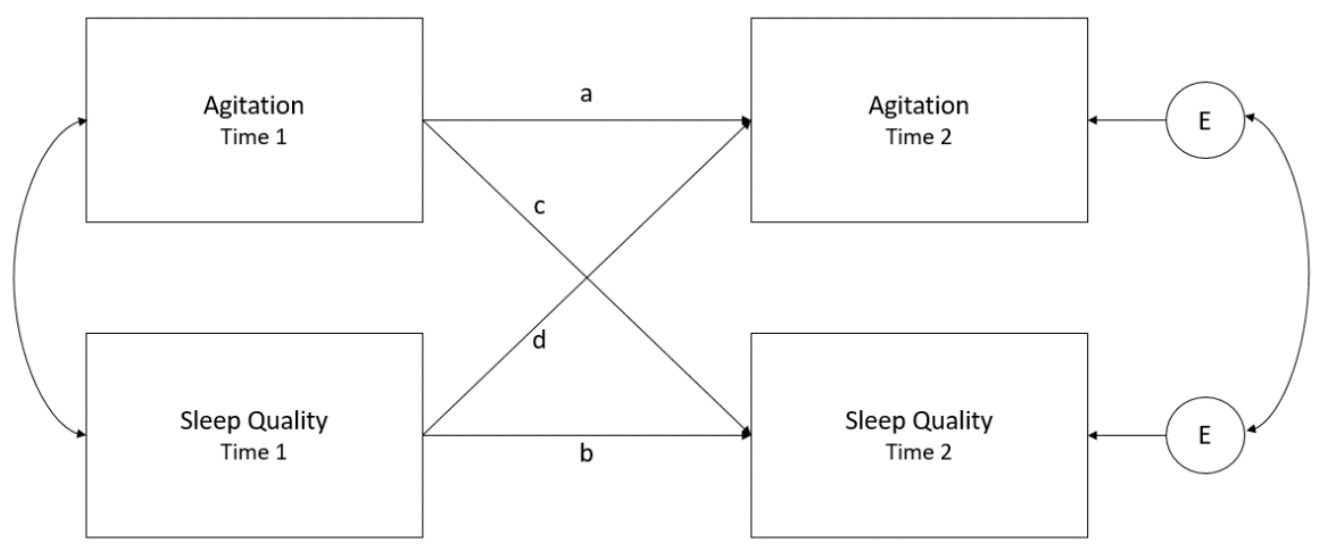

Figure 2. Cross-lagged panel model of agitation and sleep quality. 


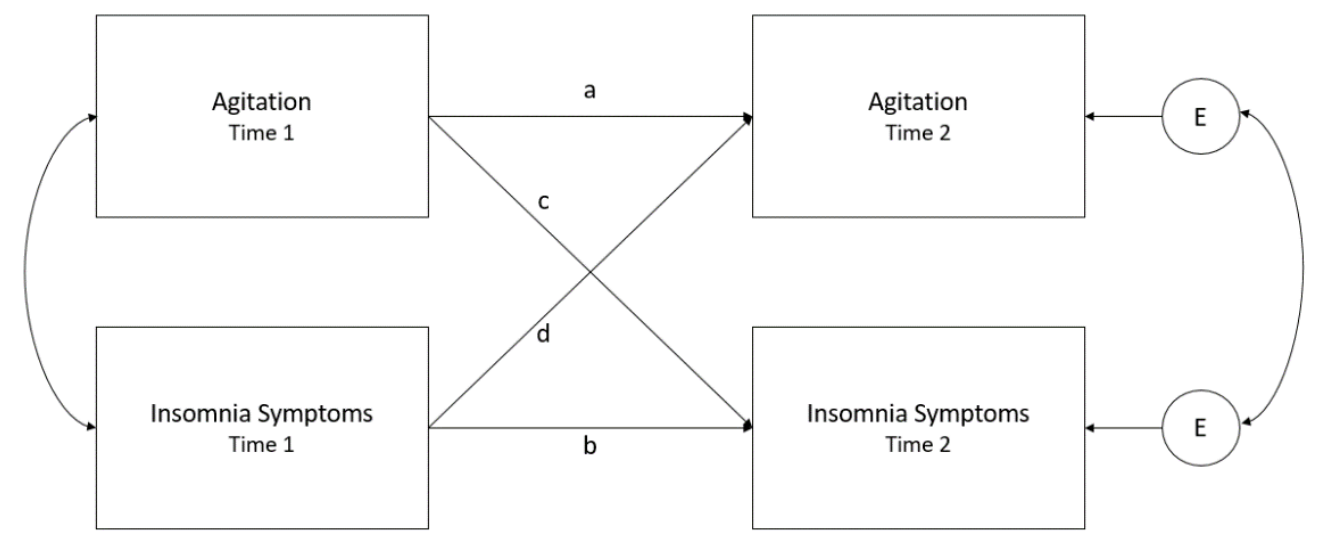

Figure 3. Cross-lagged panel model of agitation and insomnia symptoms. 


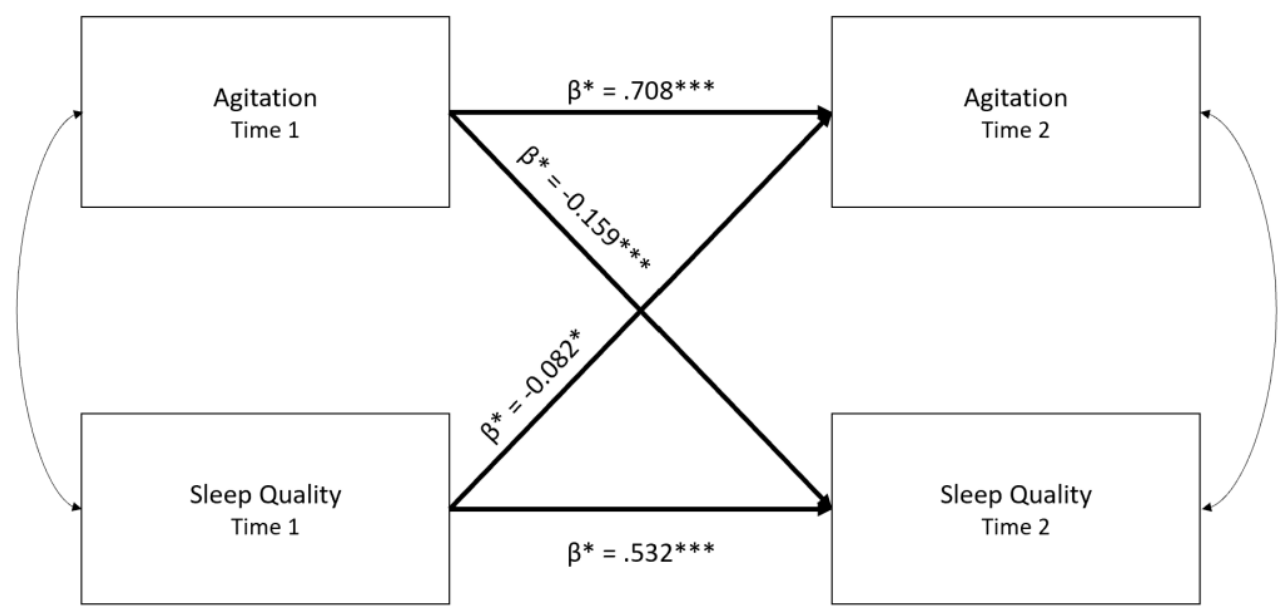

Figure 4. Results of cross-lagged panel model of agitation and sleep quality. ${ }^{*} p<.05$, $* * * p<.001$. 


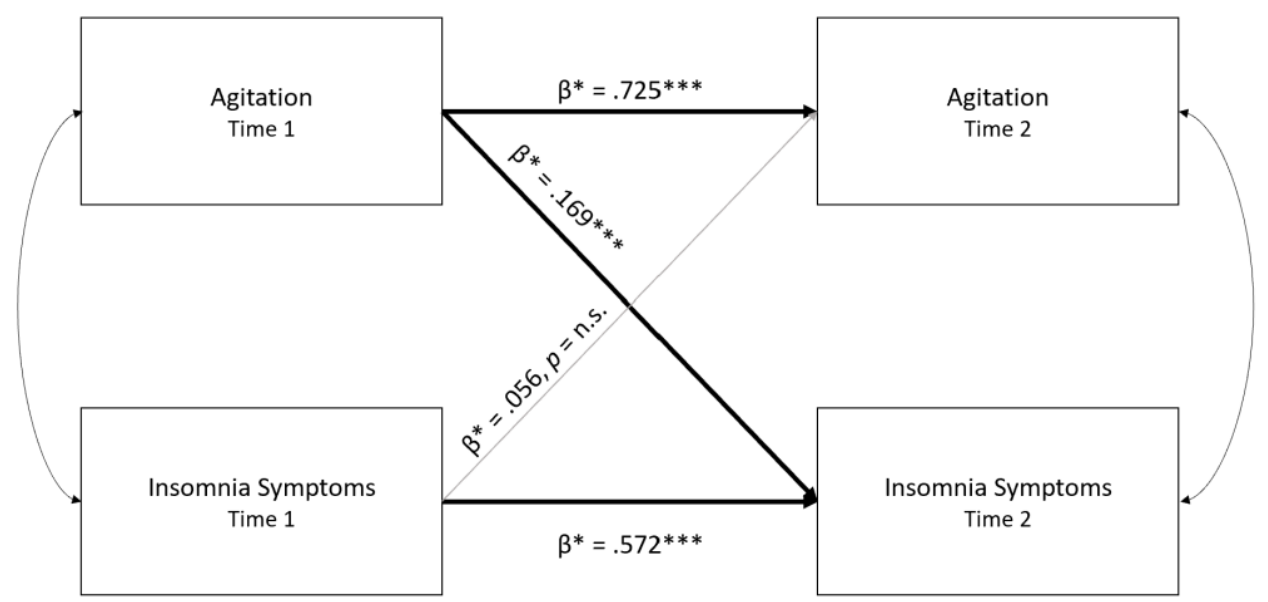

Figure 5. Results of cross-lagged panel model of agitation and insomnia symptoms. $* * * p<.001$. 


\section{References}

Ahern, J., Worthen, M., Masters, J., Lippman, S. A., Ozer, E. J., \& Moos, R. (2015). The challenges of Afghanistan and Iraq veterans' transition from military to civilian life and approaches to reconnection. PLoS ONE, 10(7), 1-13. https://doi.org/10.1371/journal.pone.0128599

Alvaro, P. K., Roberts, R. M., \& Harris, J. K. (2013). A Systematic Review Assessing Bidirectionality between Sleep Disturbances, Anxiety, and Depression. Sleep, 36(7), 1059-1068. https://doi.org/10.5665/sleep.2810

Barcikowski, R. S. (1981). Statistical Power with Group Mean as the Unit of Analysis. Journal of Educational Statistics, 6(3), 267-285.

Bernert, R. A., Joiner, T. E., Cukrowicz, K. C., Schmidt, N. B., \& Krakow, B. (2005). Suicidality and sleep disturbances. Sleep, 28(9), 1135-1141. https://doi.org/10.1093/sleep/28.9.1135

Bernert, R. A., Kim, J. S., Iwata, N. G., \& Perlis, M. L. (2015). Sleep Disturbances as an Evidence-Based Suicide Risk Factor. Current Psychiatry Reports, 17(3), 1-9. https://doi.org/10.1007/s11920-015-0554-4

Bernert, R. A., Turvey, C. L., Conwell, Y., \& Joiner, T. E. (2014). Association of poor subjective sleep quality with risk for death by suicide during a 10-year period a longitudinal, population-based study of late life. JAMA Psychiatry, 71(10), 11291137. https://doi.org/10.1001/jamapsychiatry.2014.1126

Blanchard, M., \& Farber, B. A. (2018). "It is never okay to talk about suicide": Patients' reasons for concealing suicidal ideation in psychotherapy. Psychotherapy Research, 
1-13. https://doi.org/10.1080/10503307.2018.1543977

Boukouvalas, E., El-Den, S., Murphy, A. L., Salvador-Carulla, L., \& O’Reilly, C. L. (2019). Exploring Health Care Professionals' Knowledge of, Attitudes Towards, and Confidence in Caring for People at Risk of Suicide: a Systematic Review. Archives of Suicide Research, O(0), 1-31. https://doi.org/10.1080/13811118.2019.1586608

Bramoweth, A. D., \& Germain, A. (2013). Deployment-related insomnia in military personnel and veterans topical collection on sleep disorders. Current Psychiatry Reports, 15(10). https://doi.org/10.1007/s11920-013-0401-4

Britt, T. W. (2000). The stigma of psychological problems in a work environment: Evidence from the screening of service members returning from Bosnia. Journal of Applied Social Psychology, 30(8), 1599-1618. https://doi.org/10.1111/j.15591816.2000.tb02457.x

Britt, T. W., Greene-Shortridge, T. M., Brink, S., Nguyen, Q. B., Rath, J., Cox, A. L., Hoge, C. W., Castro, C. A., Shortridge, T. M. G., Brink, S., Nguyen, Q. B., Rath, J., Cox, A. L., Hoge, C. W., \& Castro, C. A. (2008). Perceived Stigma and Barriers to Care for Psychological Treatment: Implications for Reactions to Stressors in Different Contexts. Journal of Social and Clinical Psychology, 27(4), 317-335. https://doi.org/10.1521/jscp.2008.27.4.317

Brossoit, R. M., Crain, T. L., Leslie, J. J., Hammer, L. B., Truxillo, D. M., \& Bodner, T. E. (2018). The Effects of Sleep on Workplace Cognitive Failure and Safety. Journal of Occupational Health Psychology, 24(4), 411-422. https://doi.org/10.1037/ocp0000139 
Bryan, C. J., Hitschfeld, M. J., Palmer, B. A., Schak, K. M., Roberge, E. M., \& Lineberry, T. W. (2014). Gender differences in the association of agitation and suicide attempts among psychiatric inpatients. General Hospital Psychiatry, 36(6), 726-731. https://doi.org/10.1016/j.genhosppsych.2014.09.013

Bryan, C. J., Morrow, C. E., Anestis, M. D., \& Joiner, T. E. (2010). A preliminary test of the interpersonal-psychological theory of suicidal behavior in a military sample. Personality and Individual Differences, 48(3), 347-350. https://doi.org/10.1016/j.paid.2009.10.023

Busch, K. A., Fawcett, J., \& Jacobs, D. G. (2003). Clinical correlates of inpatient suicide. Journal of Clinical Psychiatry, 64(1), 14-19. https://doi.org/10.4088/JCP.v64n0105 Buysse, D.J., Reynolds, C.F., Monk, T.H., Berman, S.R., \& Kupfer, D. J. (1998). The Pittsburgh Sleep Quality Index: a new instrument for psychiatric practice and research. In Psychiatry Research (Vol. 28, pp. 193-213).

Cacioppo, J. T., Hawkley, L. C., Berntson, G. G., Ernst, J. M., Gibbs, A. C., Stickgold, R., \& Hobson, J. A. (2002). Do lonely days invade the nights? Potential social modulation of sleep efficiency. Psychological Science, 13(4), 384-387. https://doi.org/10.1111/j.0956-7976.2002.00469.x

Centers for Disease Control and Prevention. (2018). Suicide rising across the US. 1-4. Chakravorty, S., Grandner, M. A., Mavandadi, S., Perlis, M. L., Sturgis, E. B., \& Oslin, D. W. (2014). Suicidal ideation in Veterans misusing alcohol: Relationships with insomnia symptoms and sleep duration. Addictive Behaviors, 39(2), 399-405. https://doi.org/10.1016/j.addbeh.2013.09.022 
Choi, N. G., DiNitto, D. M., Marti, C. N., \& Kaplan, M. S. (2017). Older Suicide Decedents: Intent Disclosure, Mental and Physical Health, and Suicide Means. American Journal of Preventive Medicine, 53(6), 772-780. https://doi.org/10.1016/j.amepre.2017.07.021

Crain, T. L., Hammer, L. B., Bodner, T. E., Kossek, E. E., Moen, P., Lilienthal, R., \& Buxton, O. M. (2014). Work-Family Conflict, Family-Supportive Supervisor Behaviors (FSSB), and Sleep Outcomes. Journal of Occupational Health Psychology, 19(2), 155-167. https://doi.org/10.1037/a0036010

Cukrowicz, K. C., Otamendi, A., Pinto, J. Y., Bernert, R. A., Krakow, B., \& Joiner, T. E. (2006). The impact of insomnia and sleep disturbances on depression and suicidality. Dreaming, 16(1), 1-10. https://doi.org/10.1037/1053-0797.16.1.1

Demers, A. (2011). When veterans return: The role of community in reintegration. Journal of Loss and Trauma, 16(2), 160-179. https://doi.org/10.1080/15325024.2010.519281

Dew, M. A., Hoch, C. C., Buysse, D. J., Monk, T. H., Begley, A. E., Houck, P. R., Hall, M., Kupfer, D. J., \& Reynolds, C. F. (2003). Healthy older adults' sleep predicts allcause mortality at 4 to 19 years of follow-up. Psychosomatic Medicine, 65(1), 6373. https://doi.org/10.1097/01.PSY.0000039756.23250.7C

Durkheim, E. (1897). Suicide: A Study in Sociology.

Fisher, K., Houtsma, C., Assavedo, B. L., Green, B. A., \& Anestis, M. D. (2017). Agitation as a Moderator of the Relationship Between Insomnia and Current Suicidal Ideation in the Military. Archives of Suicide Research, 21(4), 531-543. 
AGITATION AND SLEEP DISTURBANCES

https://doi.org/10.1080/13811118.2016.1193077

Gottlieb, D. J., Redline, S., Nieto, F. J., Baldwin, C. M., Newman, A. B., Resnick, H. E., \& Punjabi, N. M. (2006). Association of usual sleep duration with hypertension: The Sleep Heart Health Study. Sleep, 29(8), 1009-1014. https://doi.org/10.1093/sleep/29.8.1009

Greene-Shortridge, T. M., Britt, T. W., \& Castro, C. A. (2007). The stigma of mental health problems in the military. Military Medicine, 172(2), 157-161. https://doi.org/10.7205/milmed.172.2.157

Hammer, L. B., Wan, W. H., Brockwood, K. J., Bodner, T., \& Mohr, C. D. (2019). Supervisor support training effects on veteran health and work outcomes in the civilian workplace. Journal of Applied Psychology, 104(1), 52-69. https://doi.org/10.1037/ap10000354

Harvey, A. G., Stinson, K., Whitaker, K. L., Moskovitz, D., \& Virk, H. (2008). The subjective meaning of sleep quality: A comparison of individuals with and without insomnia. Sleep, 31(3), 383-393. https://doi.org/10.1093/sleep/31.3.383

Hoge, C., Castro, C., Messer, S. C., McGurk, D., Cotting, D. I., \& Koffman, R. L. (2004). Combat Duty in Iraq and Afganistan, Mental Health Problems, and Barriers to Care. The New England Journal of Medicine, 351(1), 13-22.

Hom, M. A., Chu, C., Schneider, M. E., Lim, I. C., Hirsch, J. K., Gutierrez, P. M., \& Joiner, T. E. (2017). Thwarted belongingness as an explanatory link between insomnia symptoms and suicidal ideation: Findings from three samples of military service members and veterans. Journal of Affective Disorders, 209(October 2016), 
AGITATION AND SLEEP DISTURBANCES

114-123. https://doi.org/10.1016/j.jad.2016.11.032

Hom, M. A., Hames, J. L., Bodell, L. P., Buchman-Schmitt, J. M., Chu, C., Rogers, M. L., Chiurliza, B., Michaels, M. S., Ribeiro, J. D., Nadorff, M. R., Winer, E. S., Lim, I. C., Rudd, M. D., \& Joiner, T. E. (2017). Investigating insomnia as a crosssectional and longitudinal predictor of loneliness: Findings from six samples. Psychiatry Research, 253(September 2016), 116-128. https://doi.org/10.1016/j.psychres.2017.03.046

Hom, M. A., Lim, I. C., Stanley, I. H., Chiurliza, B., Podlogar, M. C., Michaels, M. S., Buchman-Schmitt, J. M., Silva, C., Ribeiro, J. D., \& Joiner, T. E. (2016). Insomnia brings soldiers into mental health treatment, predicts treatment engagement, and outperforms other suicide-related symptoms as a predictor of major depressive episodes. Journal of Psychiatric Research, 79(May), 108-115. https://doi.org/10.1016/j.jpsychires.2016.05.008

Hom, M. A., Stanley, I. H., Podlogar, M. C., \& Joiner, T. E. (2017). “Are You Having Thoughts of Suicide?" Examining Experiences With Disclosing and Denying Suicidal Ideation. Journal of Clinical Psychology, 73(10), 1382-1392. https://doi.org/10.1002/jclp.22440

Husky, M. M., Zablith, I., Alvarez Fernandez, V., \& Kovess-Masfety, V. (2016). Factors associated with suicidal ideation disclosure: Results from a large population-based study. Journal of Affective Disorders, 205, 36-43. https://doi.org/10.1016/j.jad.2016.06.054

Kenny, D. A. (1975). Cross-lagged panel correlation: A test for spuriousness. 
AGITATION AND SLEEP DISTURBANCES

Psychological Bulletin, 82(6), 887-903. https://doi.org/10.1037/0033-2909.82.6.887

Kenny, D. A. (2005). Cross-Lagged Panel Design. In B. S. Everitt \& D. C. Howell (Eds.), Encyclopedia of Statistics in Behavioral Science (pp. 450-451). https://doi.org/10.1002/9781118445112.stat06464

Kenny, D. A., \& Harackiewicz, J. M. (1979). Cross-lagged panel correlation: Practice and promise. Journal of Applied Psychology, 64(4), 372-379. https://doi.org/10.1037/0021-9010.64.4.372

Kent, R. G., Uchino, B. N., Cribbet, M. R., Bowen, K., \& Smith, T. W. (2015). Social Relationships and Sleep Quality. Annals of Behavioral Medicine, 49(6), 912-917. https://doi.org/10.1007/s12160-015-9711-6

Kessler, R. C., Berglund, P., Nock, M., Wang, P. S., \& Page, P. (2005). Trends in Suicide Ideation, Plans, Gestures, and Attempts in the United States, 1990-1992 to 20012003. Journal of the American Medical Association, 293(20), 2487-2495.

Kodaka, M., Matsumoto, T., Katsumata, Y., Akazawa, M., Tachimori, H., Kawakami, N., Eguchi, N., Shirakawa, N., \& Takeshima, T. (2014). Suicide risk among individuals with sleep disturbances in Japan: A case-control psychological autopsy study. Sleep Medicine, 15(4), 430-435. https://doi.org/10.1016/j.sleep.2013.11.789

Kovasznay, B., Miraglia, R., Beer, R., \& Way, B. (2004). Reducing suicides in New York state correctional facilities. Psychiatric Quarterly, 75(1), 61-70. https://doi.org/10.1023/B:PSAQ.0000007561.83444.a4

Krakow, B., Ribeiro, J. D., Ulibarri, V. A., Krakow, J., \& Joiner, T. E. (2011). Sleep disturbances and suicidal ideation in sleep medical center patients. Journal of 
AGITATION AND SLEEP DISTURBANCES

Affective Disorders, 131(1-3), 422-427. https://doi.org/10.1016/j.jad.2010.12.001

Michail, M., Tait, L., \& Churchill, D. (2017). General practitioners' clinical expertise in managing suicidal young people: Implications for continued education. Primary Health Care Research and Development, 18(5), 419-428.

https://doi.org/10.1017/S1463423617000299

Muthén, L. K., \& Muthén, B. (2019). The Comprehensive Nodelling Program for Applied Researchers: user's guide.

Newsom, J. T. (2016). Cross-Lagged Panel Analysis. In The Encyclopedia of Adulthood and Aging (pp. 1-6). https://doi.org/10.1002/9781118528921.wbeaa250

Office of Mental Health and Suicide Prevention. (2018). VA National Suicide Data Report 2005-2016. https://www.mentalhealth.va.gov/docs/datasheets/OMHSP_National_Suicide_Data_Report_2005-2016_508-compliant.pdf

Pietrzak, R. H., Goldstein, M. B., Malley, J. C., Rivers, A. J., Johnson, D. C., \& Southwick, S. M. (2010). Risk and protective factors associated with suicidal ideation in veterans of Operations Enduring Freedom and Iraqi Freedom. Journal of Affective Disorders, 123(1-3), 102-107. https://doi.org/10.1016/j.jad.2009.08.001

Pigeon, W. R., Britton, P. C., Ilgen, M. A., Chapman, B., \& Conner, K. R. (2012). Sleep disturbance preceding suicide among veterans. American Journal of Public Health, 102(SUPPL. 1), 93-97. https://doi.org/10.2105/AJPH.2011.300470

Resnick, H. E., Redline, S., Shahar, E., Gilpin, A., Newman, A., Walter, R., Ewy, G. a, Howard, B. V, \& Punjabi, N. M. (2003). Diabetes and Sleep Disturbances: Findings from the Sleep Heart Health Study. Diabetes Care, 26(3), 702-709. 
AGITATION AND SLEEP DISTURBANCES

https://doi.org/10.2337/diacare.26.3.702

Ribeiro, J. D., Bender, T. W., Selby, E. A., Hames, J. L., \& Joiner, T. E. (2011).

Development and validation of a brief self-report measure of agitation: The brief agitation measure. Journal of Personality Assessment, 93(6), 597-604.

https://doi.org/10.1080/00223891.2011.608758

Ribeiro, J. D., Bodell, L. P., Hames, J. L., Hagan, C. R., \& Joiner, T. E. (2013). An

empirically based approach to the assessment and management of suicidal behavior.

Journal of Psychotherapy Integration, 23(3), 207-221.

https://doi.org/10.1037/a0031416

Ribeiro, J. D., Silva, C., \& Joiner, T. E. (2014). Overarousal interacts with a sense of fearlessness about death to predict suicide risk in a sample of clinical outpatients.

Psychiatry Research, 218(1-2), 106-112.

https://doi.org/10.1016/j.psychres.2014.03.036

Rogers, M. L., \& Joiner, T. E. (2018). Severity of Suicidal Ideation Matters:

Reexamining Correlates of Suicidal Ideation Using Quantile Regression. Journal of Clinical Psychology, 74(3), 442-452. https://doi.org/10.1002/jclp.22499

Rogers, M. L., Schneider, M. E., Tucker, R. P., Law, K. C., Anestis, M. D., \& Joiner, T. E. (2017). Overarousal as a mechanism of the relation between rumination and suicidality. Journal of Psychiatric Research, 92, 31-37. https://doi.org/10.1016/j.jpsychires.2017.03.024

Rogers, M. L., Stanley, I. H., Hom, M. A., Chiurliza, B., Podlogar, M. C., \& Joiner, T. E. (2018). Conceptual and Empirical Scrutiny of Covarying Depression Out of Suicidal 
AGITATION AND SLEEP DISTURBANCES

Ideation. Assessment, 25(2), 159-172. https://doi.org/10.1177/1073191116645907

Rogers, M. L., Tucker, R. P., Law, K. C., Michaels, M. S., Anestis, M. D., \& Joiner, T. E. (2016). Manifestations of overarousal account for the association between cognitive anxiety sensitivity and suicidal ideation. Journal of Affective Disorders, 192, 116124. https://doi.org/10.1016/j.jad.2015.12.014

Rudd, D. M., Berman, A. L., Joiner, T. E., Nock, M. K., Silverman, M. M., Mandrusiak, M., Van Orden, K., \& Witte, T. (2006). Warning signs for suicide: Theory, research, and clinical applications. Suicide and Life-Threatening Behavior, 36(3), 255-262. https://doi.org/10.1521/suli.2006.36.3.255

Sani, G., Tondo, L., Koukopoulos, A., Reginaldi, D., Kotzalidis, G. D., Koukopoulos, A. E., Manfredi, G., Mazzarini, L., Pacchiarotti, I., Simonetti, A., Ambrosi, E., Angeletti, G., Girardi, P., \& Tatarelli, R. (2011). Suicide in a large population of former psychiatric inpatients. Psychiatry and Clinical Neurosciences, 65(3), 286295. https://doi.org/10.1111/j.1440-1819.2011.02205.x

Selby, E. A., Anestis, M. D., Bender, T. W., Ribeiro, J. D., Nock, M. K., Rudd, M. D., Bryan, C. J., Lim, I. C., Baker, M. T., Gutierrez, P. M., \& Joiner, T. E. (2010). Overcoming the fear of lethal injury: Evaluating suicidal behavior in the military through the lens of the Interpersonal-Psychological Theory of Suicide. Clinical Psychology Review, 30(3), 298-307. https://doi.org/10.1016/j.cpr.2009.12.004

Silva, C., Smith, A. R., Dodd, D. R., Covington, D. W., \& Joiner, T. E. (2016). Suiciderelated knowledge and confidence among behavioral health care staff in seven states. Psychiatric Services, 67(11), 1240-1245. 
AGITATION AND SLEEP DISTURBANCES

https://doi.org/10.1176/appi.ps.201500271

Troxel, W. M., Shih, R. a, Pedersen, E., Geyer, L., Fisher, M. P., Griffin, B. A., Haas, A. C., Kurz, J. R., \& Steinberg, P. S. (2015). Sleep in the Military: Promoting Healthy Sleep Among U.S. Servicemembers. RAND Health Quarterly, 5(2).

Van Orden, K. A., Witte, T. K., Cukrowicz, K. C., Braithwaite, S. R., Selby, E. A., \& Joiner, T. E. (2010). The Interpersonal Theory of Suicide. Psychological Review, 117(2), 575-600. https://doi.org/10.1037/a0018697

Xu, J., Murphy, S. L., Kochanek, K. D., Bastian, B., \& Arias, E. (2018). Death: Final Report for 2016. National Vital Statistics Report, 67(2), 1-76. 


\section{Appendix}

\section{Survey Items}

\section{Agitation}

Brief Agitation Measure (BAM; Ribeiro et al., 2011)

Instructions: Please rate the extent to which each of the following statements have been true for you in the past week.

$1=$ Not at all true, $7=$ Certainly true

1. I want to crawl out of my skin.

2. I feel so stirred up inside I want to scream.

3. I feel a lot of emotional turmoil in my gut.

\section{Sleep Behavior}

Selected Items from the Pittsburg Sleep Quality Index (PSQI; Buysse et al., 1998) Instructions: The following questions relate to your usual sleep habits during the past month. Your answers should indicate the most accurate reply for the majority of days and nights in the PAST 30 DAYS.

Sleep Quality

During the PAST 30 DAYS, how would you rate your sleep quality overall?

$1=$ Very bad, 2 = Fairly bad, $3=$ Fairly good, $4=$ Very good

\section{Insomnia Symptoms}

1. During the PAST 30 DAYS, how often could you not get to sleep within 30 minutes?

2. During the PAST 30 DAYS, how often did you wake in the middle of the night or early morning?

$1=$ Never, $2=$ Less than once a week, $3=$ Once or twice a week, $4=3+$ times a week

\section{Psychological Distress}

K6; Kessler et al., 2002

Instructions: Thinking about the PAST MONTH, about how often did you...

$1=$ None of the time, $2=$ A little of the time, $3=$ Some of the time, $4=$ Most of the time

1. Feel so depressed that nothing could cheer you up?

2. Feel hopeless?

3. Feel restless or fidgety?

4. Feel that everything was an effort?

5. Feel worthless?

6. Feel nervous? 


\section{Demographics}

What is your gender?

What is your age?

What is your current marital status?

Single

In a committed relationship (not cohabitating)

Cohabitating (not married)

Married

Divorced

Separated

Widowed

Civil commitment or union

How many children do you have?

What is the highest degree or level of school you have completed?

Are you of Hispanic, Latino or Spanish Origin?

What is your race/ethnicity? (select all that apply)

American Indian or Alaska Native

Asian

Black or African American

Native Hawaiian or Pacific Islander

Non-Hispanic White

Other

What best describes your current military status?

Active

Separated

What is your present or most recent Service Branch/Military Component?

Army National Guard

Air National Guard

Army Reserves

Marine Reserves

Navy Reserves

Air Force Reserves

Coast Guard Reserves

Army

Navy

Air Force

Marine

Coast Guard

How long have you been or were you in the military?

What is your present or most recent rank/pay grade?

Number of times deployed including foreign and domestic post 9/11? 